\title{
Enantiopure Amidinate Complexes of the Rare-Earth Elements
}

Tobias S. Brunner, Paul Benndorf, Michael T. Gamer, Nicolai Knöfel, Katharina Gugau, and Peter W. Roesky*

Institut für Anorganische Chemie, Karlsruher Institut für Technologie (KIT), Engesserstr. 15, 76131 Karlsruhe, Germany.

AUTHOR EMAIL ADDRESS roesky@kit.edu

Supporting Information 


\section{Catalysis}

Table S1: HPLC conditions for determination of enantiomeric excess values of $\left[\{(S)-P E T A\}_{2} \operatorname{Ln}\left\{\mathrm{E}_{(}\left(\operatorname{SiMe}_{3}\right)_{2}\right\}\right]$ $(\mathrm{E}=\mathrm{N}, \mathrm{Ln}=\mathrm{Y}(\mathbf{8}) ; \mathrm{E}=\mathrm{CH}, \mathrm{Ln}=\mathrm{Sc}(\mathbf{9}), \mathrm{Y}(\mathbf{1 0}), \mathrm{Lu}(\mathbf{1 1}))$ catalyzed hydroamination reactions. $^{[\mathrm{a}]}$

\begin{tabular}{|c|c|c|c|c|c|}
\hline Entry & Compound & $\begin{array}{l}\text { Flow rate } \\
{[\mathrm{mL} / \mathrm{min}]}\end{array}$ & $\begin{array}{l}\text { Retention time [min] } \\
\text { (absolute config) }\end{array}$ & catalyst & $\begin{array}{l}e e \\
{[\%]}\end{array}$ \\
\hline 1 & & & & 8 & $15(S)$ \\
\hline 2 & & & $7.3(S)$ & 9 & $46(S)$ \\
\hline 3 & $\begin{array}{l}{ }_{\mathrm{Ph}} \mathrm{X}_{\mathrm{Pl}} \\
12 \mathrm{~b}\end{array}$ & 1.00 & $12.4(R)$ & 10 & $23(S)$ \\
\hline 4 & & & & 11 & $52(S)$ \\
\hline 5 & & & & 9 & $33(S)$ \\
\hline 6 & & 1.00 & $\begin{array}{l}6.5(S) \\
9.9(R)\end{array}$ & 10 & $17(S)$ \\
\hline 7 & $13 \mathrm{~b}$ & & & 11 & $36(S)$ \\
\hline 8 & & & $6.4(S)$ & 9 & $17(S)$ \\
\hline 9 & $\begin{array}{c}\mathrm{Me}_{14 \mathrm{~b}} \\
{ }_{14}\end{array}$ & 1.00 & $10.1(R)$ & 10 & $17(S)$ \\
\hline 10 & & & & 11 & $45(S)$ \\
\hline 11 & & & $8.3(S)$ & 9 & $\mathrm{rac}^{[\mathrm{b}]}$ \\
\hline 12 & & 1.00 & $10.4(R)$ & 10 & $\operatorname{rac}^{[b]}$ \\
\hline 13 & & & & 11 & $11(R)^{[\mathrm{b}]}$ \\
\hline 14 & & & $10.4(R)$ & 9 & rac \\
\hline 15 & $16 \mathrm{~b}$ & 0.75 & $19.3(S)$ & 10 & $5(R)$ \\
\hline 16 & & & & 11 & n.d. ${ }^{[\mathrm{c}]}$ \\
\hline
\end{tabular}

[a] Regis $(R, R)-\beta$-Gem1 column (i.d. $=4.6 \mathrm{~mm}$, length $=250 \mathrm{~mm}$, particle size $=5 \mathrm{~mm})$; $\lambda_{\max }: 254 \mathrm{~nm}$, injected volume: $1 \mu \mathrm{L}$; eluent ratio: $n$-hexane: $i \mathrm{PrOH}$ 75:25; temperature of column $25^{\circ} \mathrm{C}$, . [b] Temperature of column $10^{\circ} \mathrm{C}$. [c] No reproducible data could be obtained. 


\section{X-ray crystallographic studies}

Figure S1: Solid state structure of $(S)$-HPETA·HCl. Hydrogen atoms are omitted for clarity. Thermal ellipsoids are drawn to encompass $30 \%$ probability.

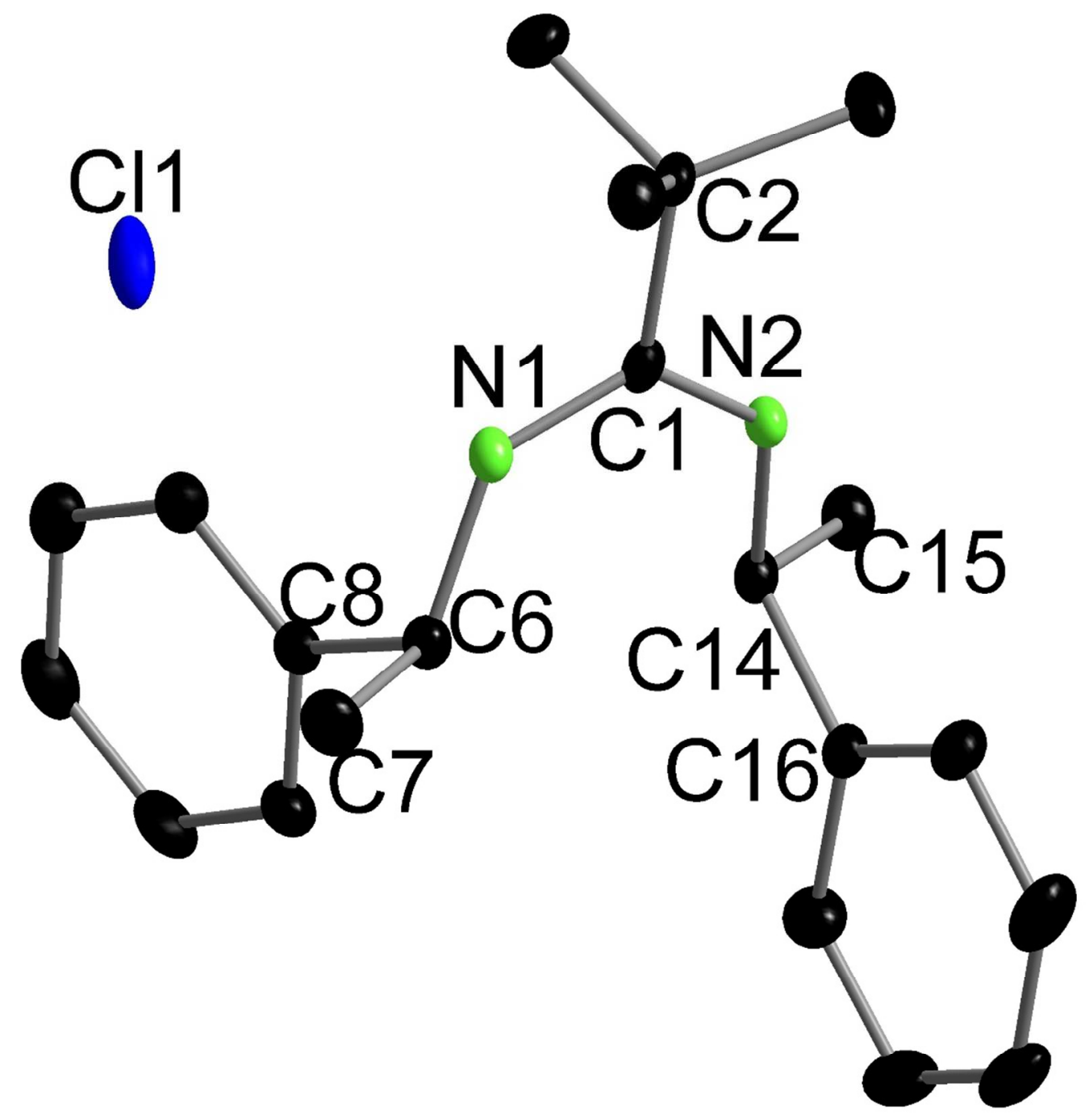


Figure S2: Solid state structure of $(\boldsymbol{S})$-LiPETA-LiCl. Hydrogen atoms are omitted for clarity. Thermal ellipsoids are drawn to encompass $30 \%$ probability.

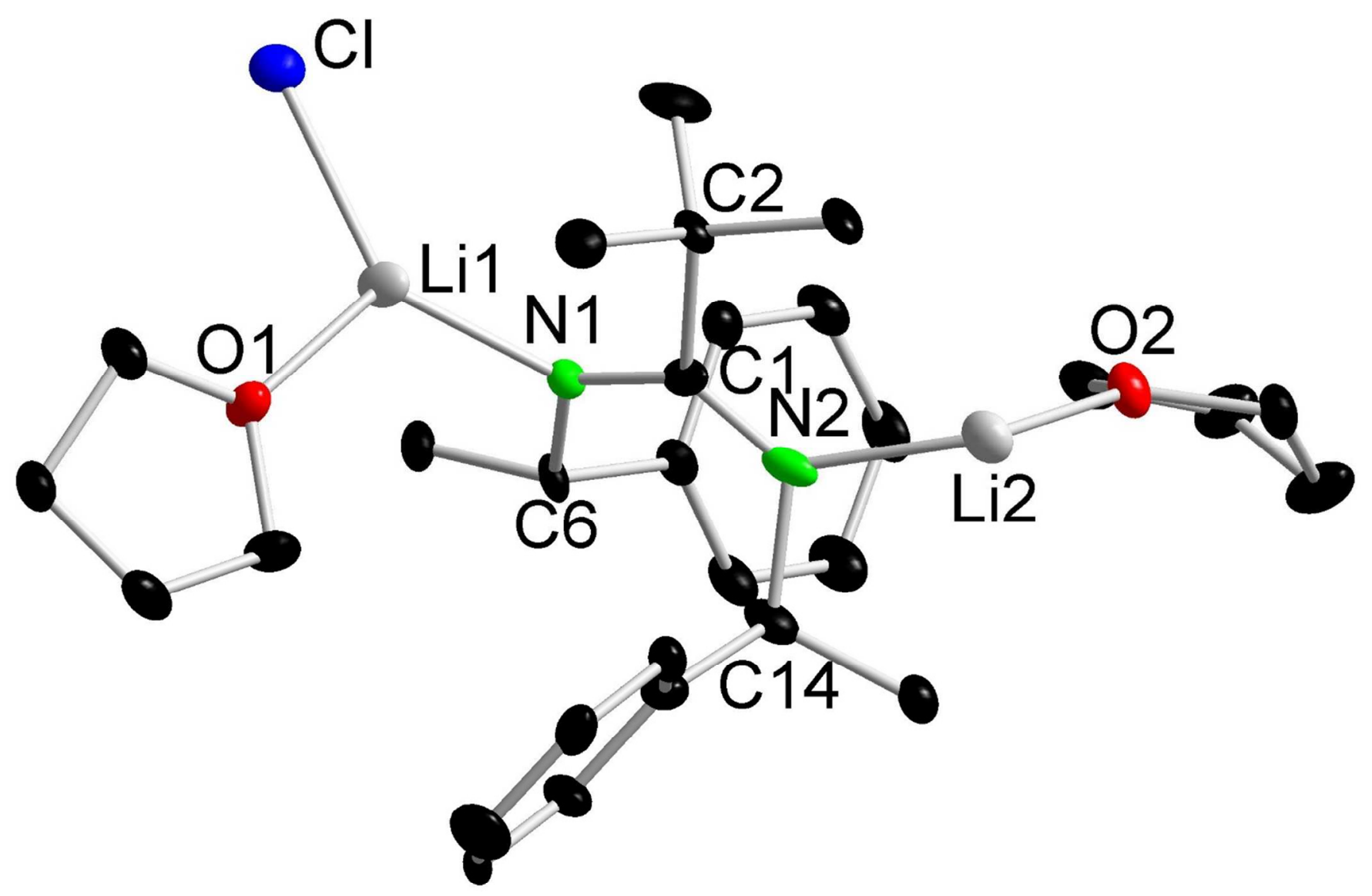


Figure S3: Solid state structure of 1. Hydrogen atoms are omitted for clarity. Thermal ellipsoids are drawn to encompass $30 \%$ probability.

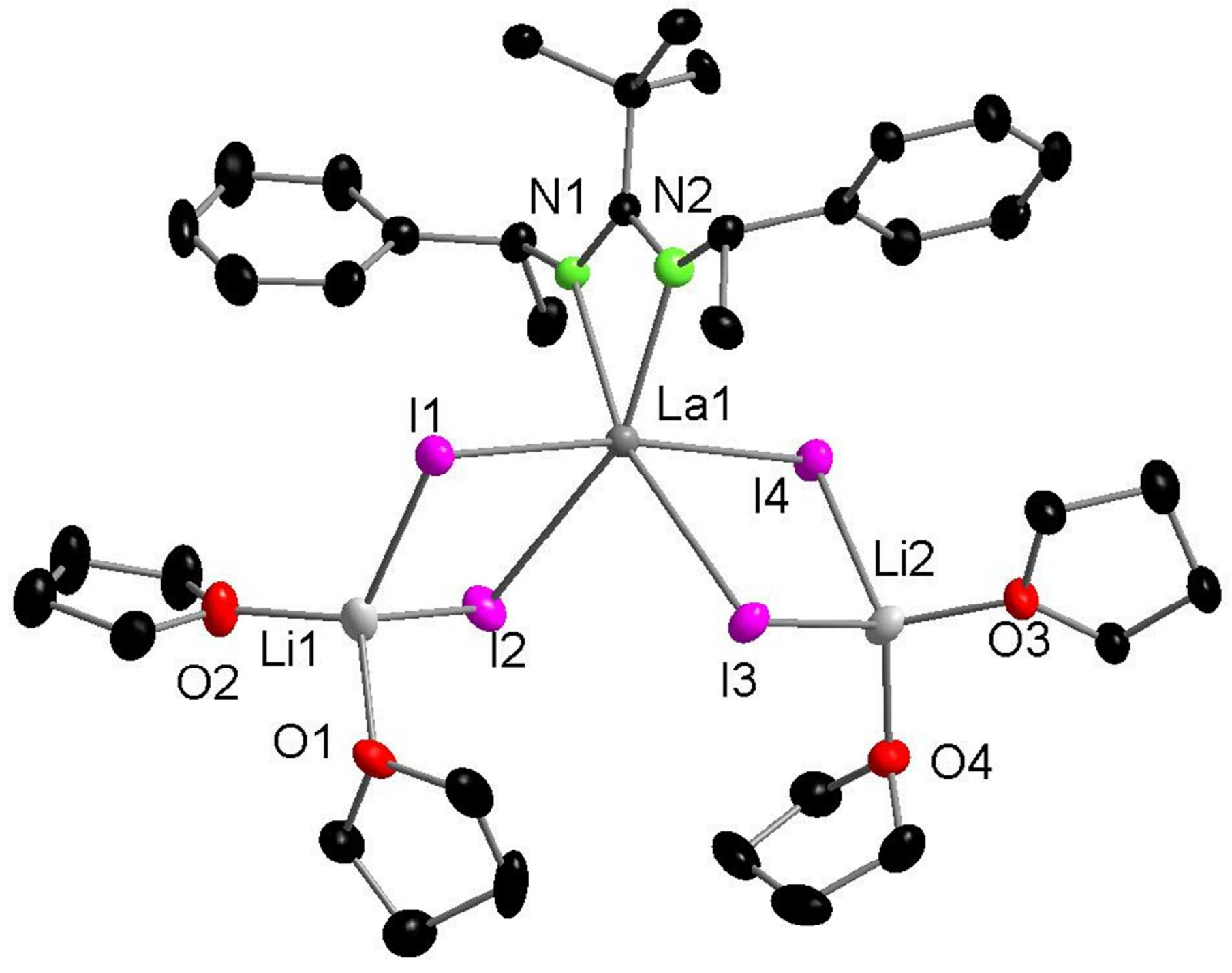


Figure S4: Solid state structure of 2. Hydrogen atoms are omitted for clarity. Thermal ellipsoids are drawn to encompass $30 \%$ probability.

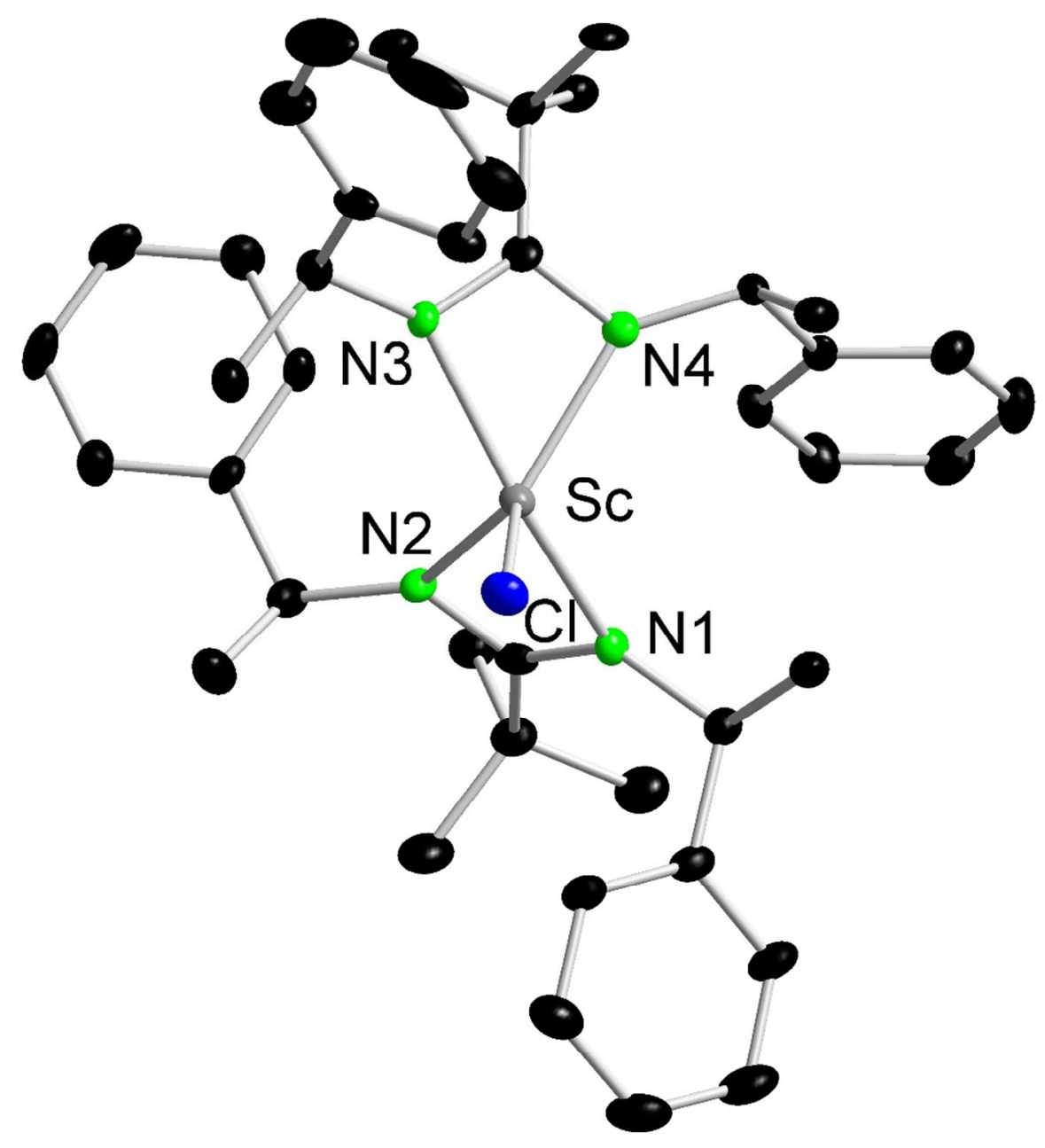


Figure S5: Solid state structure of 3. Hydrogen atoms are omitted for clarity. Thermal ellipsoids are drawn to encompass $30 \%$ probability.

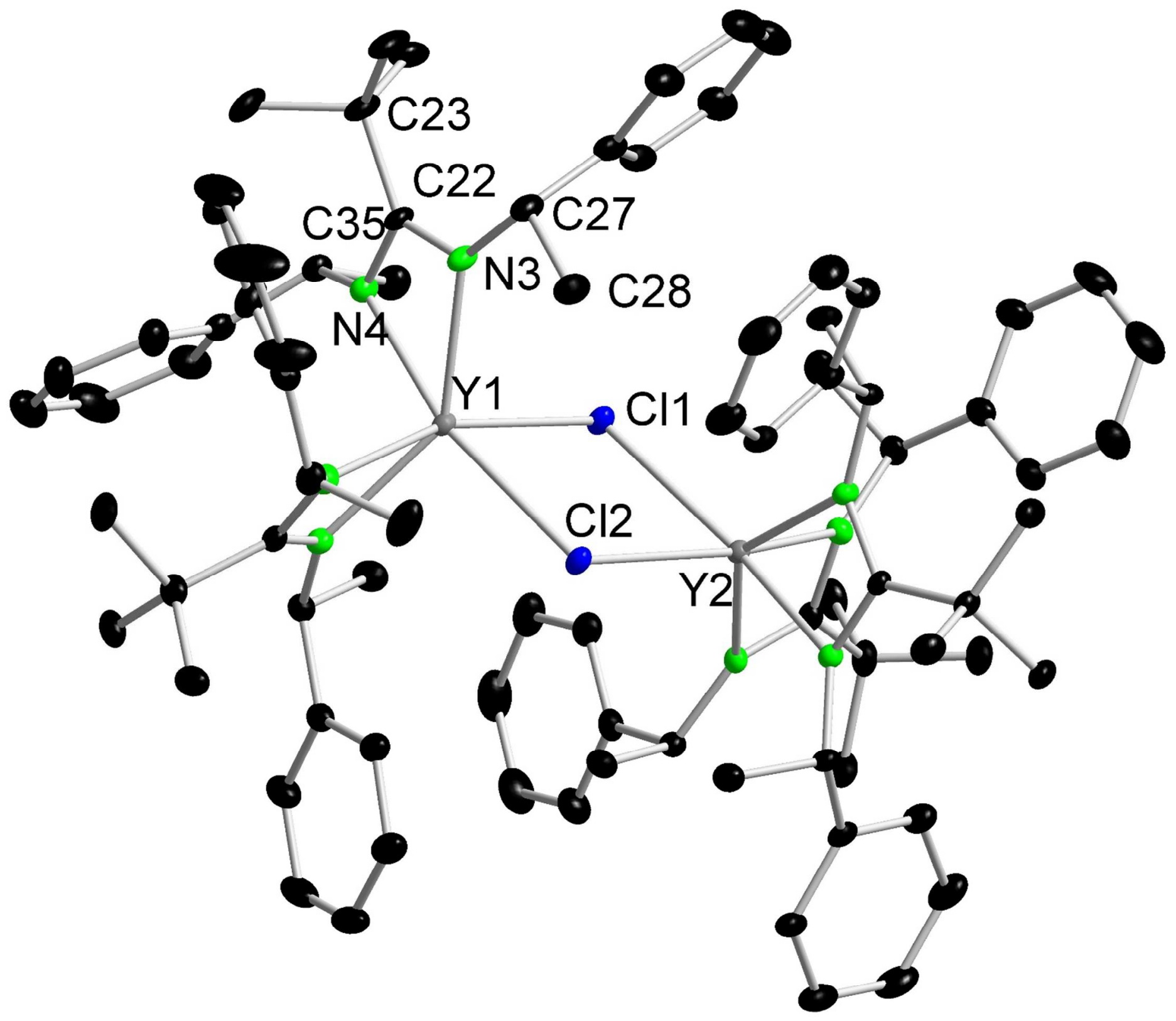


Figure S6: Solid state structure of 4. Hydrogen atoms are omitted for clarity. Thermal ellipsoids are drawn to encompass $30 \%$ probability.

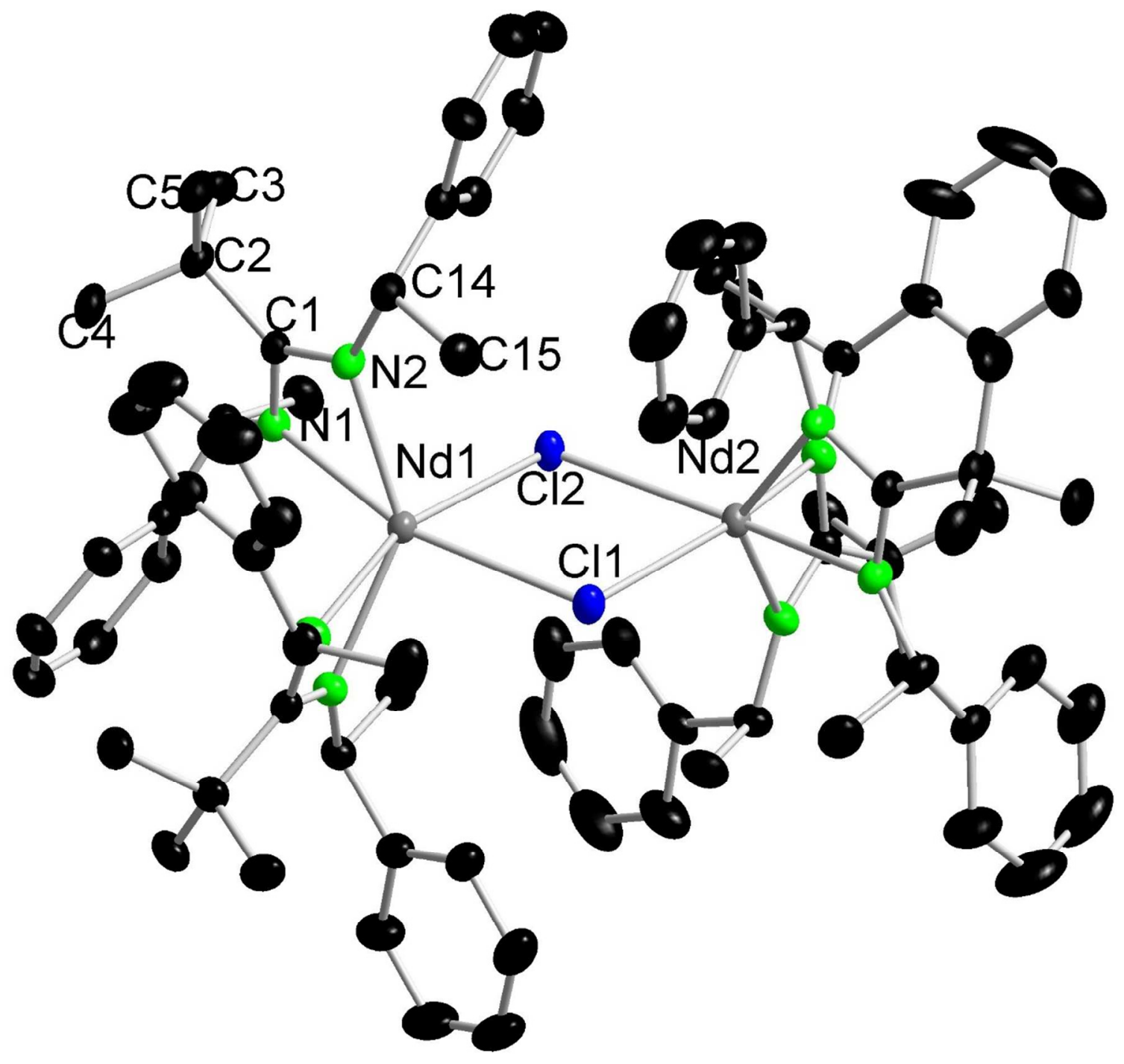


Figure S7: Solid state structure of 5. Hydrogen atoms are omitted for clarity. Thermal ellipsoids are drawn to encompass $30 \%$ probability.

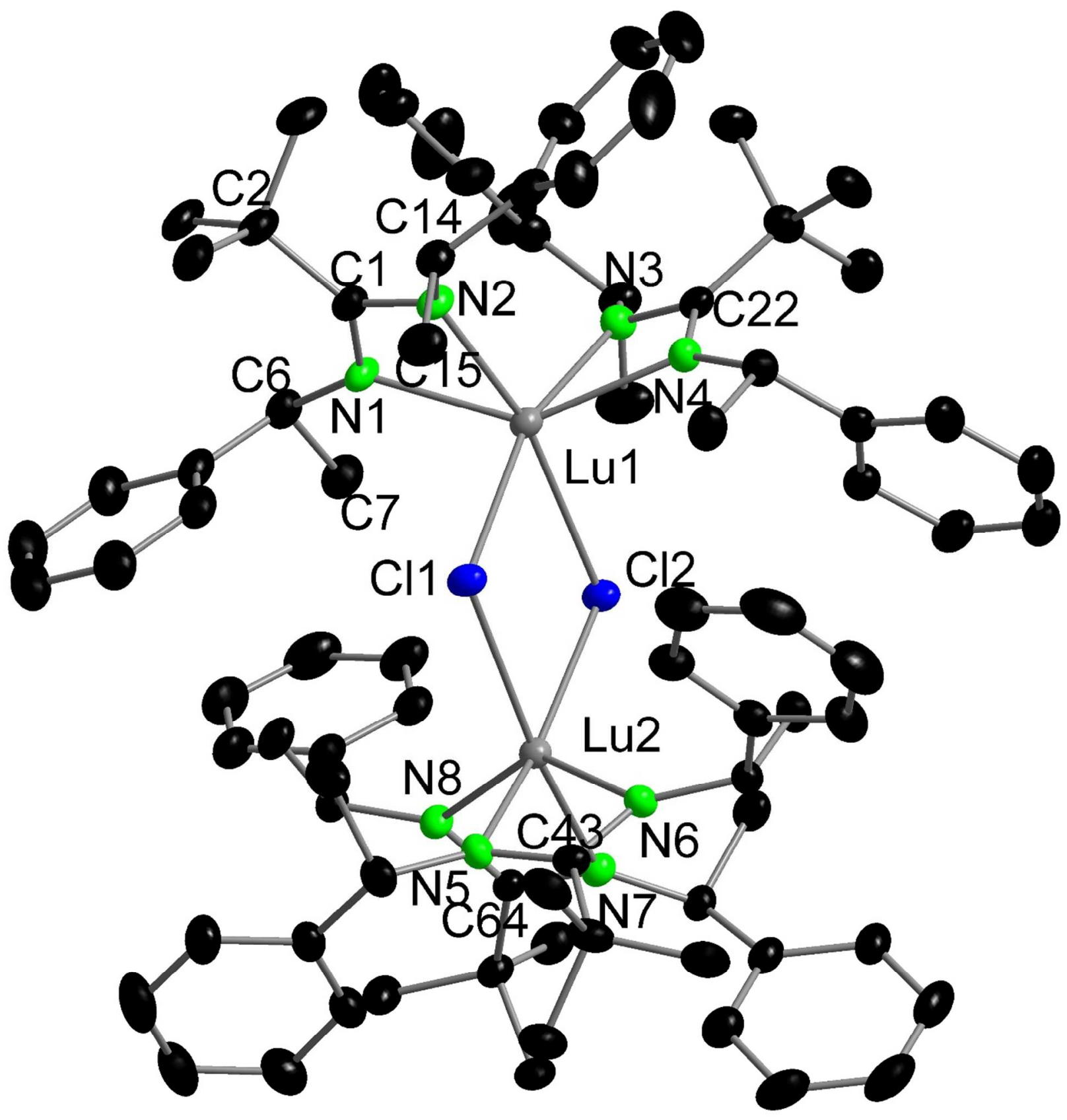


Figure S8: Solid state structure of 6. Hydrogen atoms are omitted for clarity. Thermal ellipsoids are drawn to encompass $30 \%$ probability.

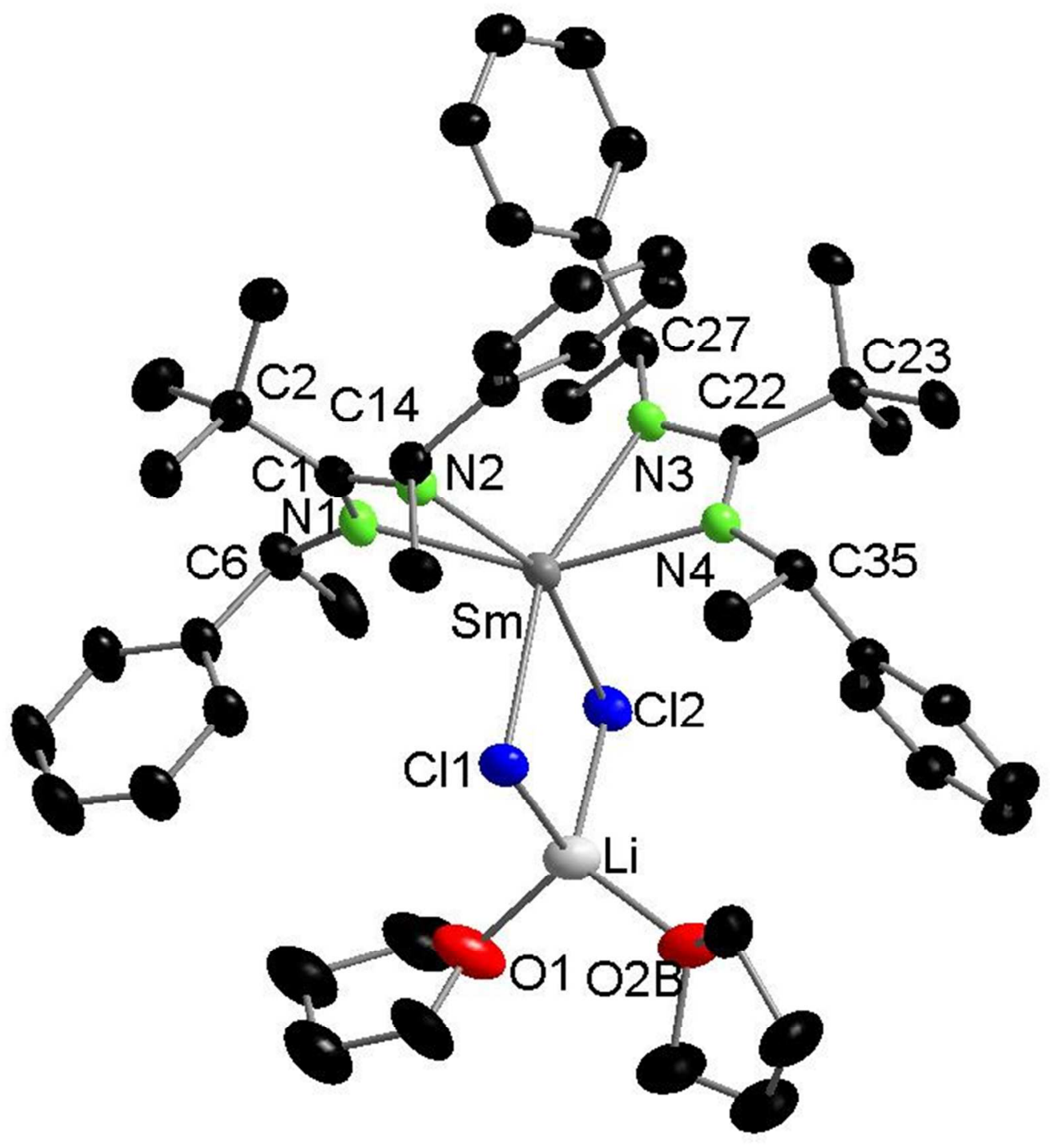


Figure S9: Solid state structure of 7. Hydrogen atoms are omitted for clarity. Thermal ellipsoids are drawn to encompass $30 \%$ probability.

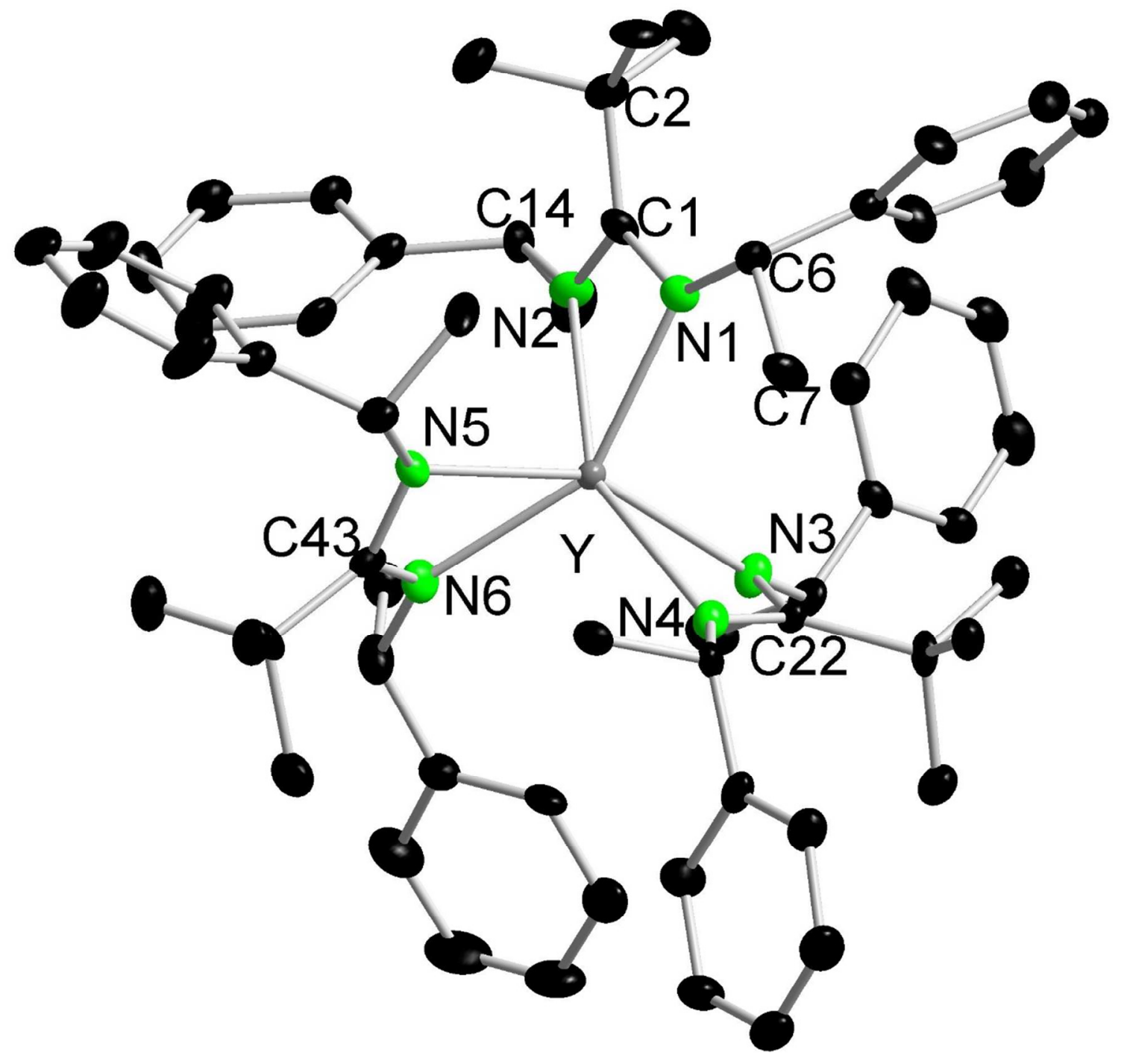


Figure S10: Solid state structure of $\mathbf{8}$. Hydrogen atoms are omitted for clarity. Thermal ellipsoids are drawn to encompass $30 \%$ probability.

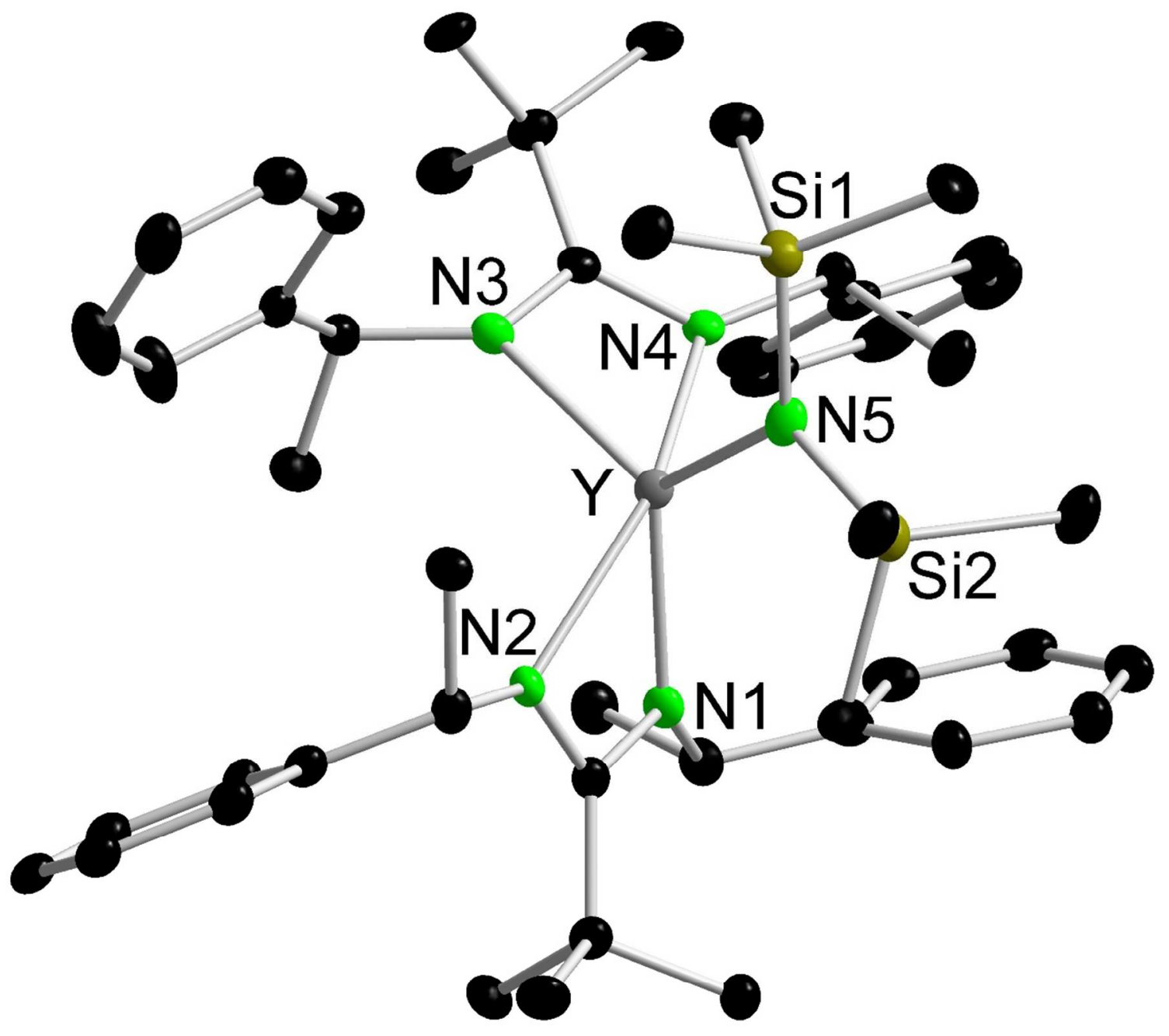


Figure S11: Solid state structure of 11. Hydrogen atoms and disorder are omitted for clarity. Only one molecule is shown. Thermal ellipsoids are drawn to encompass $30 \%$ probability.

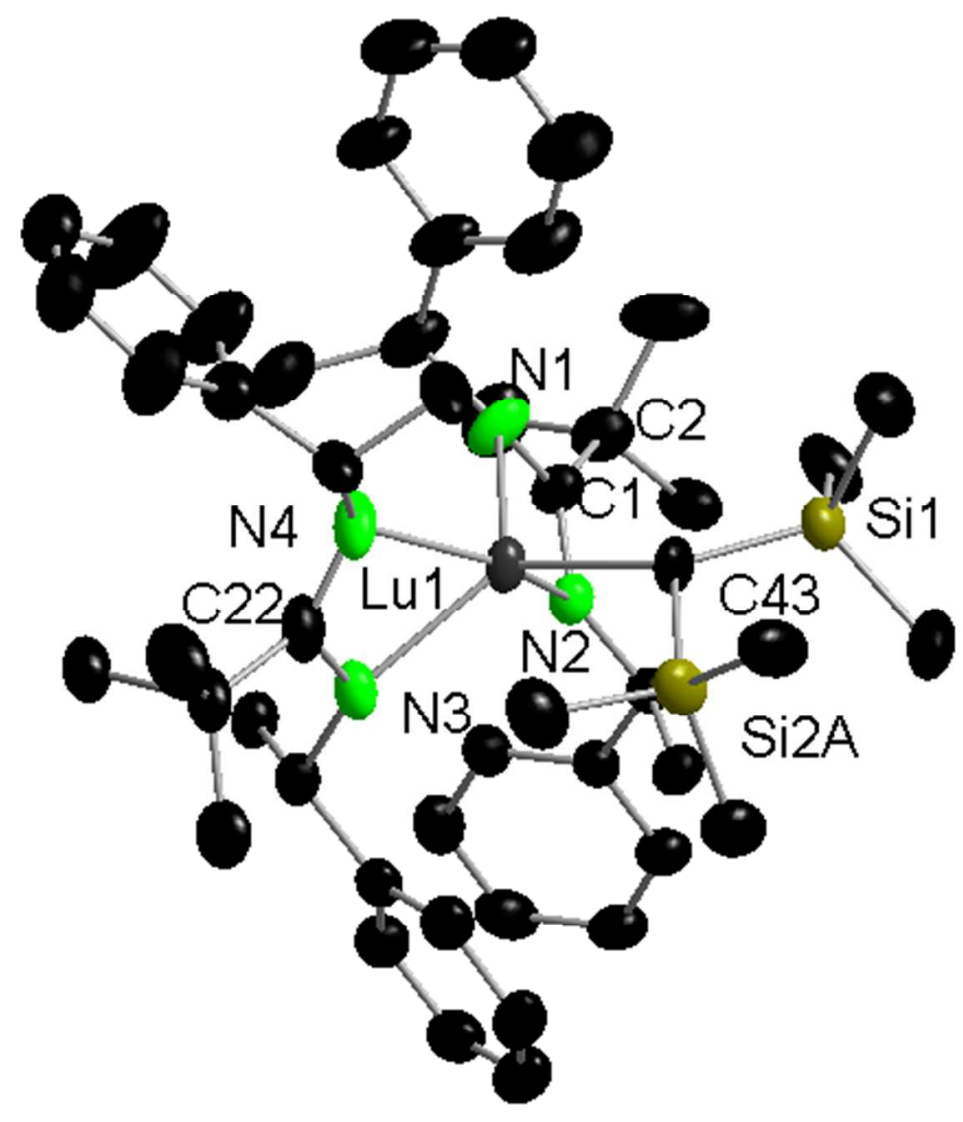


NMR

Figure S12. ${ }^{1} \mathrm{H} \mathrm{NMR}$ of I (300 MHz, $\left.\mathrm{CDCl}_{3}, 298 \mathrm{~K}\right)$.

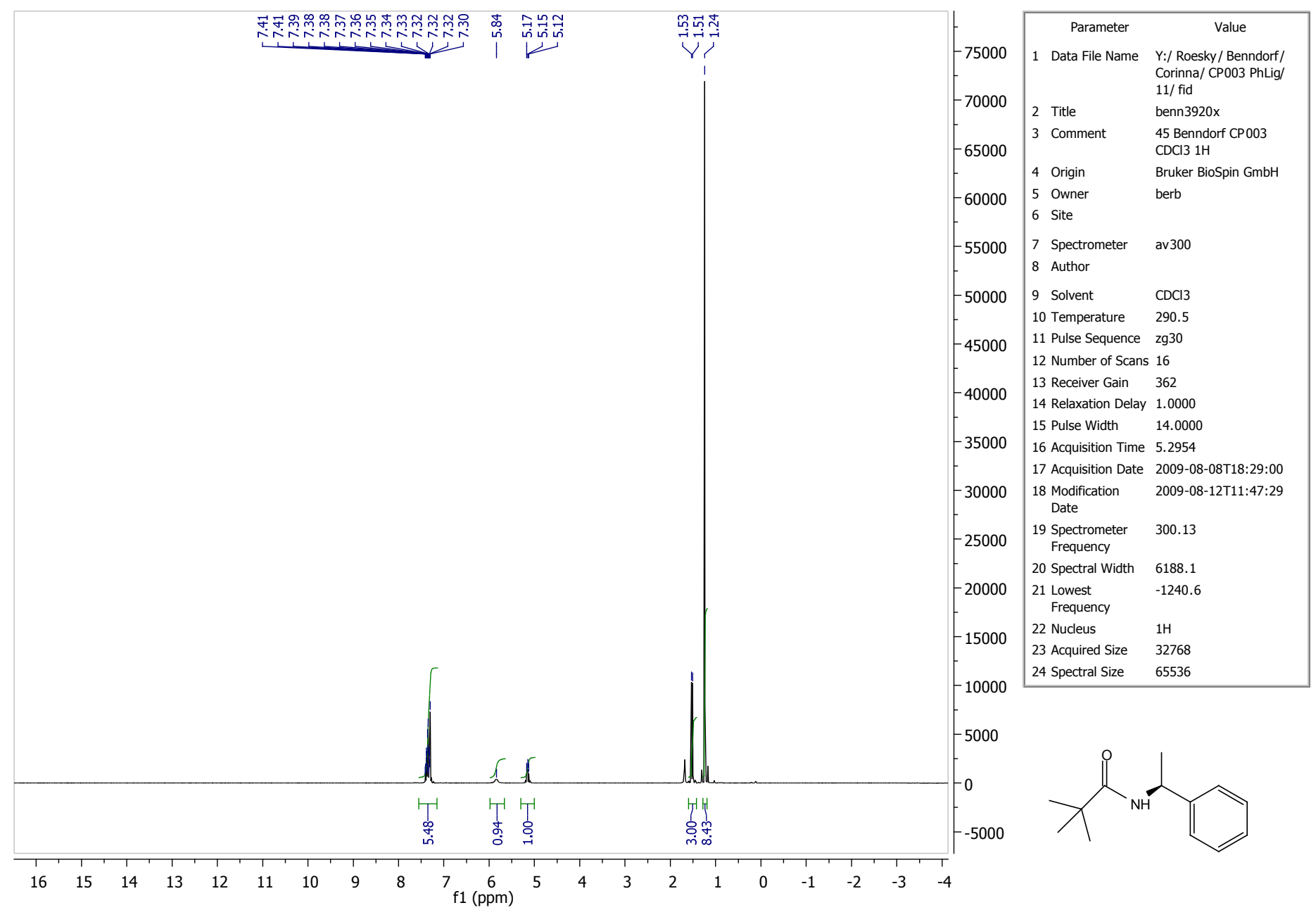


Figure S13. ${ }^{13} \mathrm{C}\left\{{ }^{1} \mathrm{H}\right\}$ NMR of $\mathbf{I}$ in $\mathrm{CDCl}_{3}\left(75 \mathrm{MHz}, \mathrm{CDCl}_{3}, 298 \mathrm{~K}\right.$, )

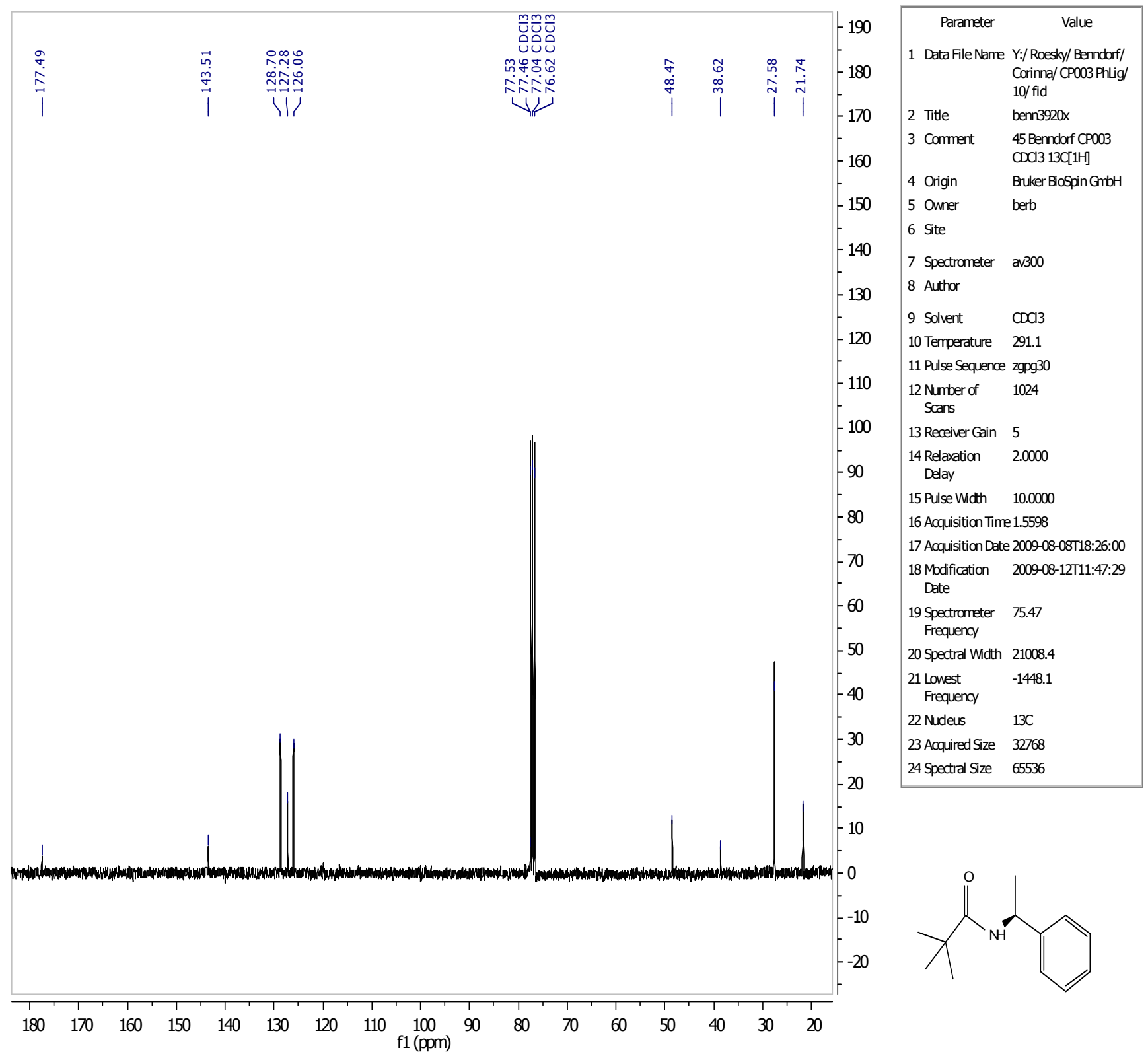


Figure S14. ${ }^{1} \mathrm{H}$ NMR of ((S)-1-Phenylethyl)carbodiimide ((S)-PEC) $\left(400 \mathrm{MHz}, \mathrm{CDCl}_{3}, 298 \mathrm{~K}\right)$.

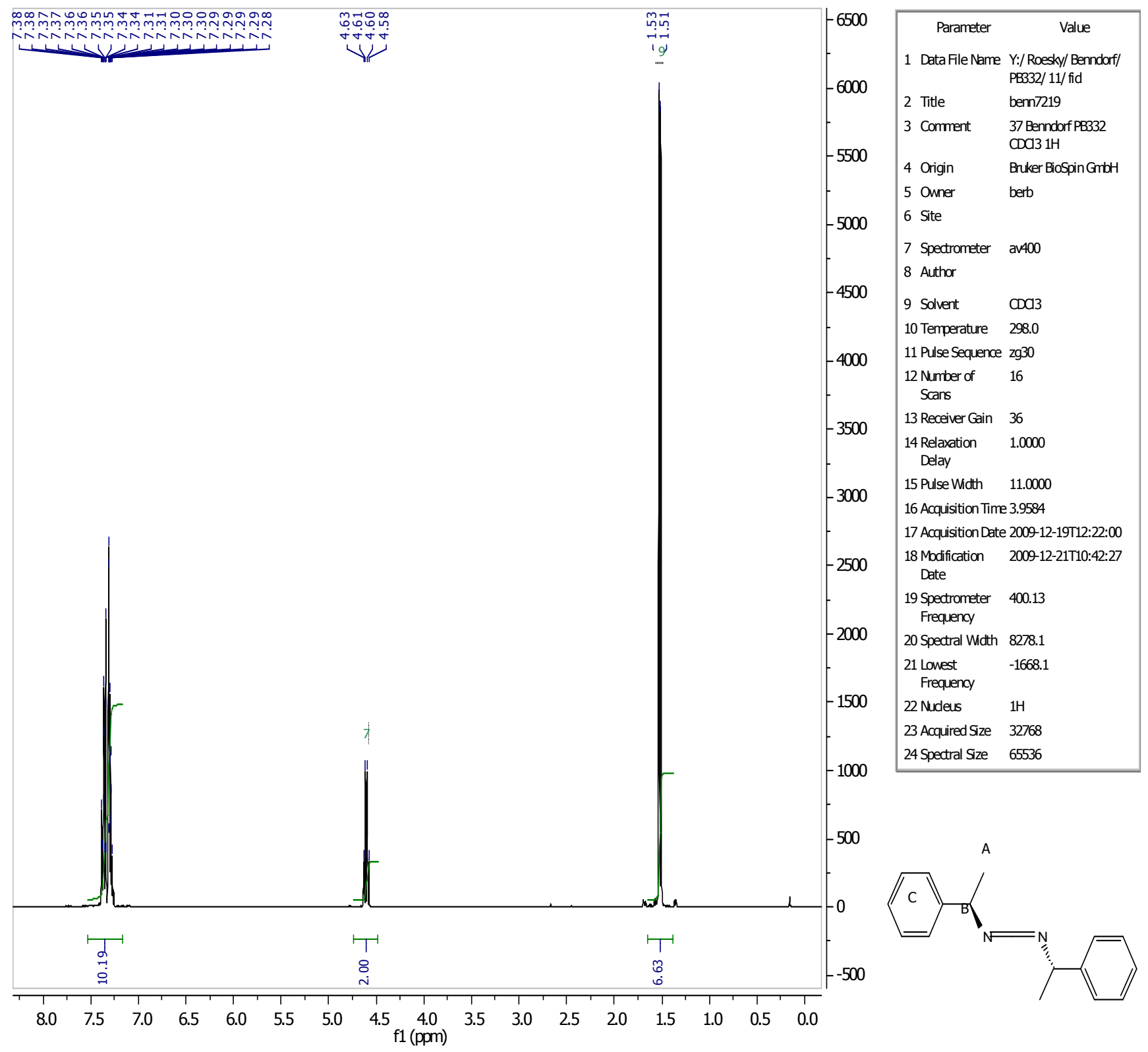


Figure S15. ${ }^{13} \mathrm{C}\left\{{ }^{1} \mathrm{H}\right\}$ NMR $((S)-1-P h e n y l e t h y l)$ carbodiimide $((S)-\mathrm{PEC})$ in $\mathrm{CDCl}_{3}\left(100 \mathrm{MHz}, \mathrm{CDCl}_{3}, 298 \mathrm{~K}\right)$.

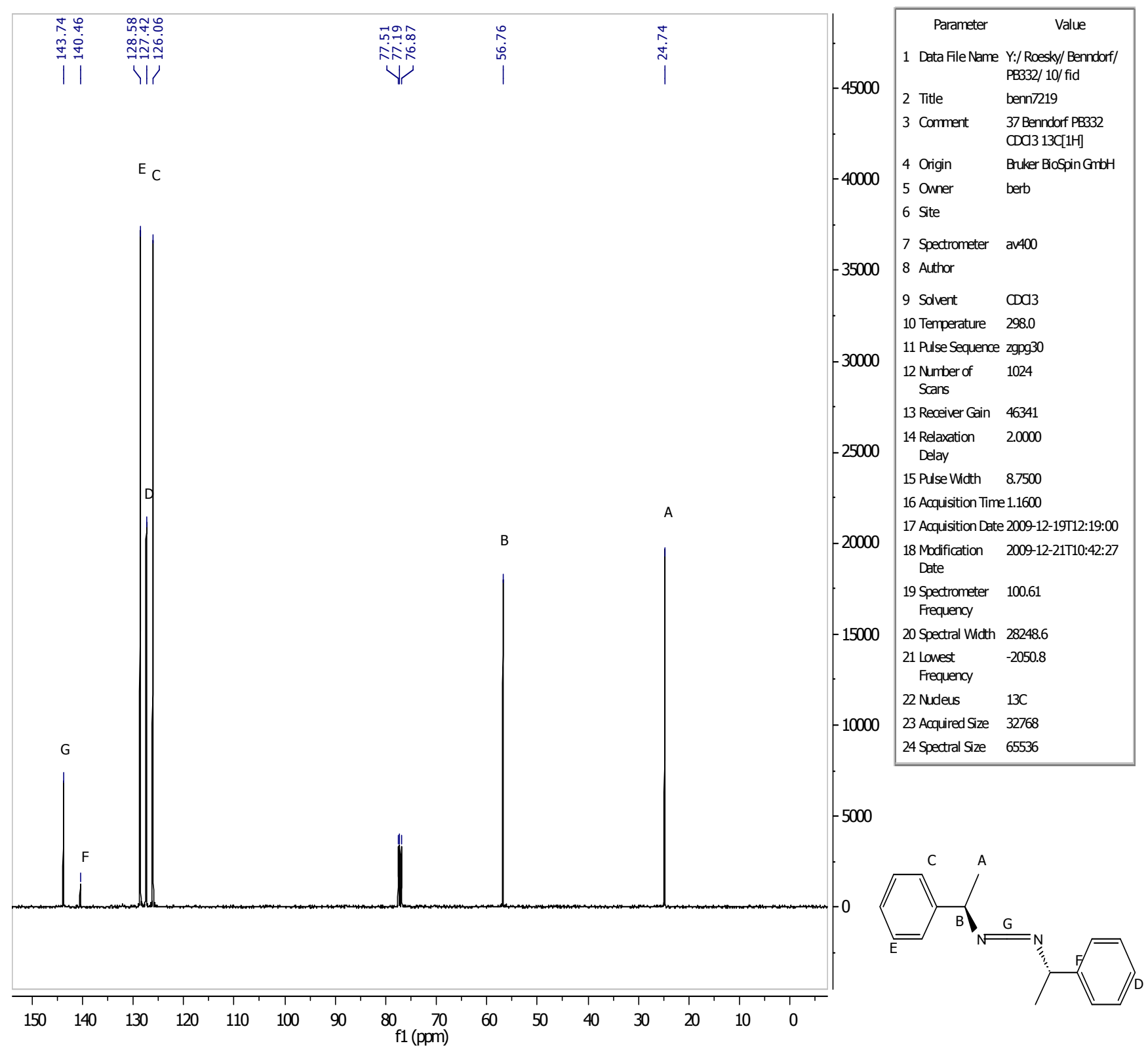


Figure S16. ${ }^{1} \mathrm{H}$ NMR of lithium-N,N'-bis-((S)-1-phenylethyl)pivalamidinate ((S)-LiPETA) (400 MHz, D8-THF, 298 K).

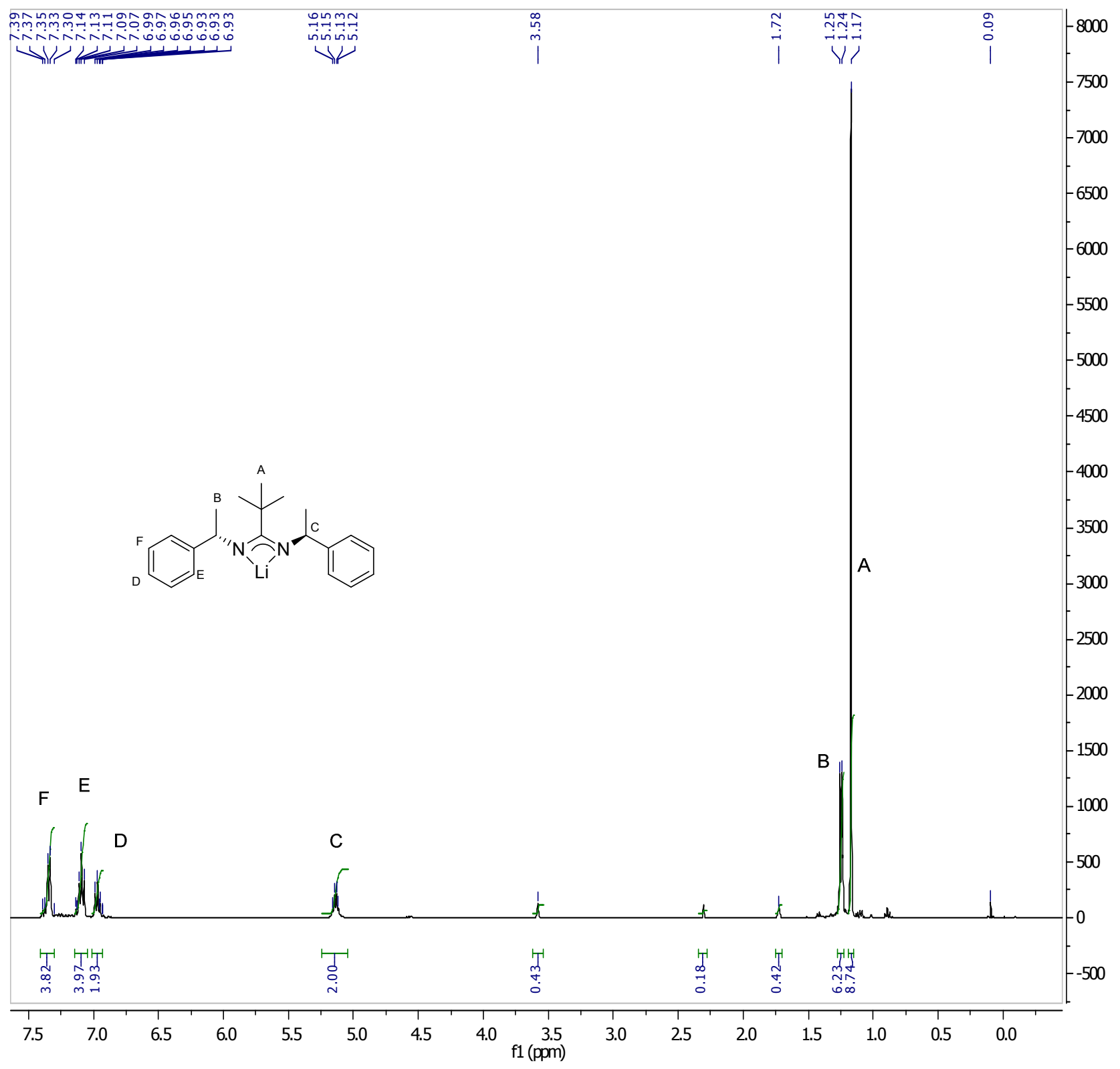


Figure S17. ${ }^{13} \mathrm{C}\left\{{ }^{1} \mathrm{H}\right\}$ NMR lithium-N,N'-bis-((S)-1-phenylethyl)pivalamidinate ((S)-LiPETA) (101MHz, D8-THF, 298 $\mathrm{K})$.

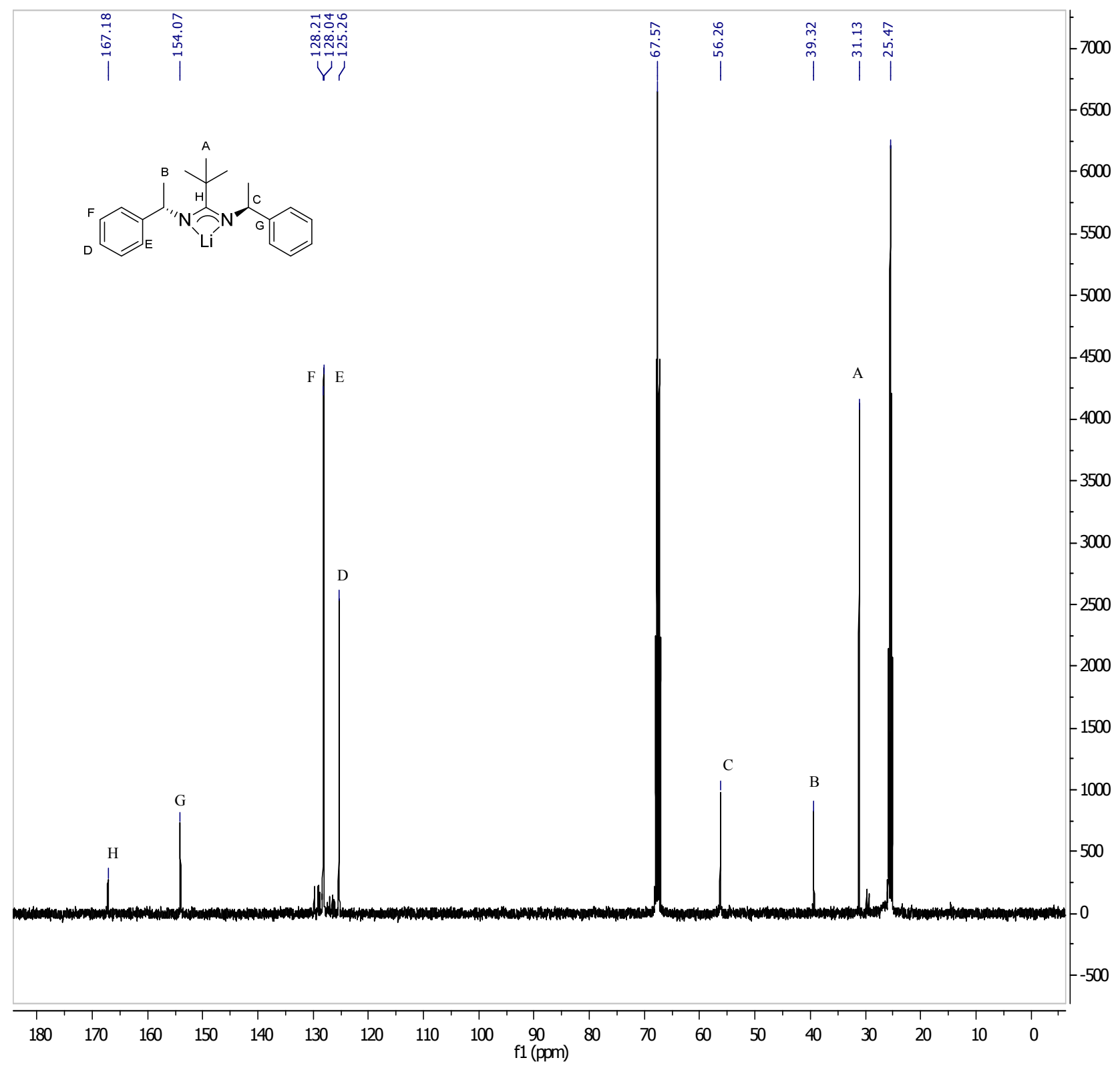


Figure S18. ${ }^{1} \mathrm{H}$ NMR of $1\left(300 \mathrm{MHz}, \mathrm{C}_{6} \mathrm{D}_{6}, 298 \mathrm{~K}\right)$.

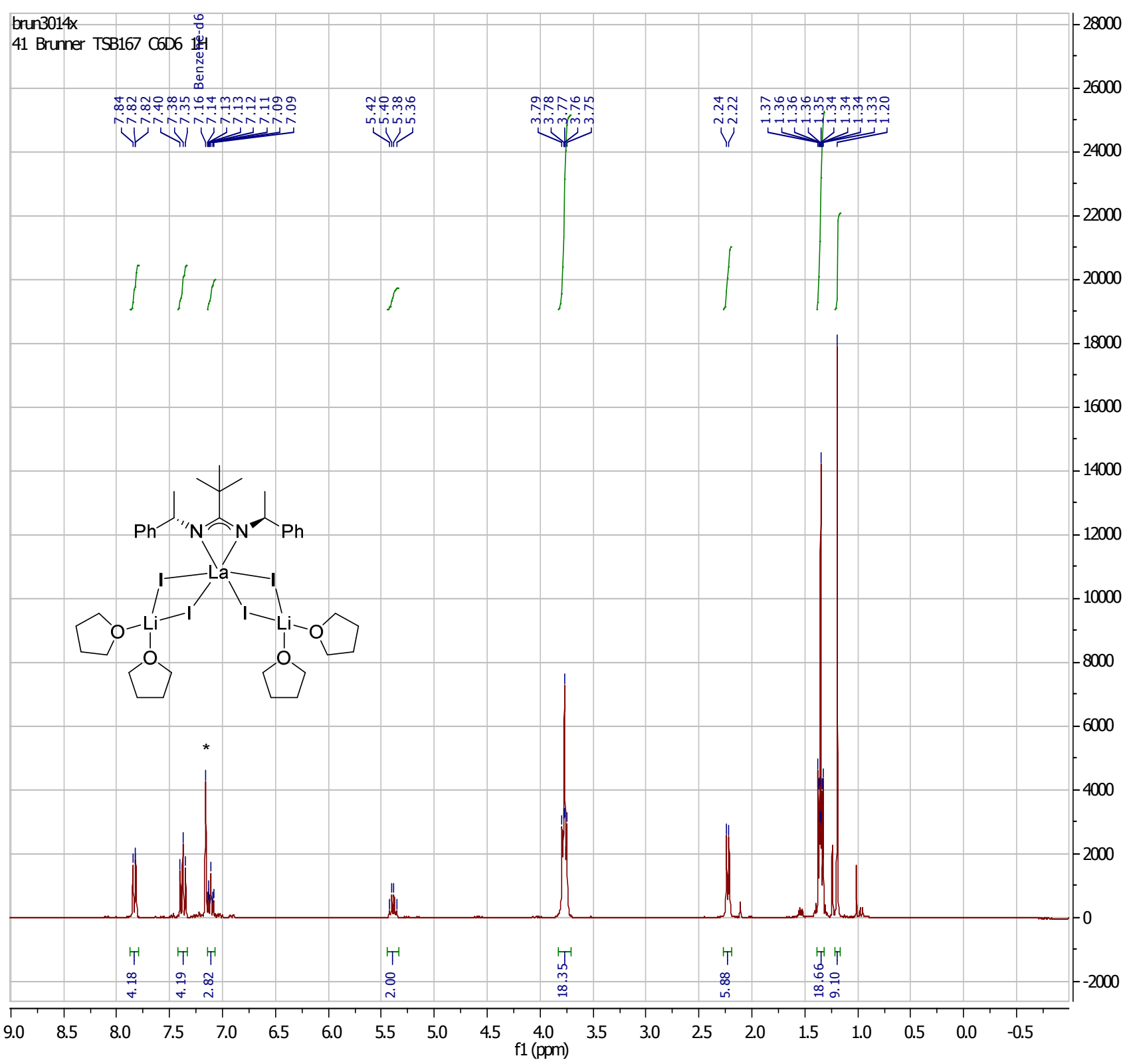

* NMR solvent (benzene) 
Figure S19. ${ }^{13} \mathrm{C}\left\{{ }^{1} \mathrm{H}\right\}$ NMR of $\mathbf{1}\left(75 \mathrm{MHz}, \mathrm{C}_{6} \mathrm{D}_{6}, 298 \mathrm{~K}\right)$.

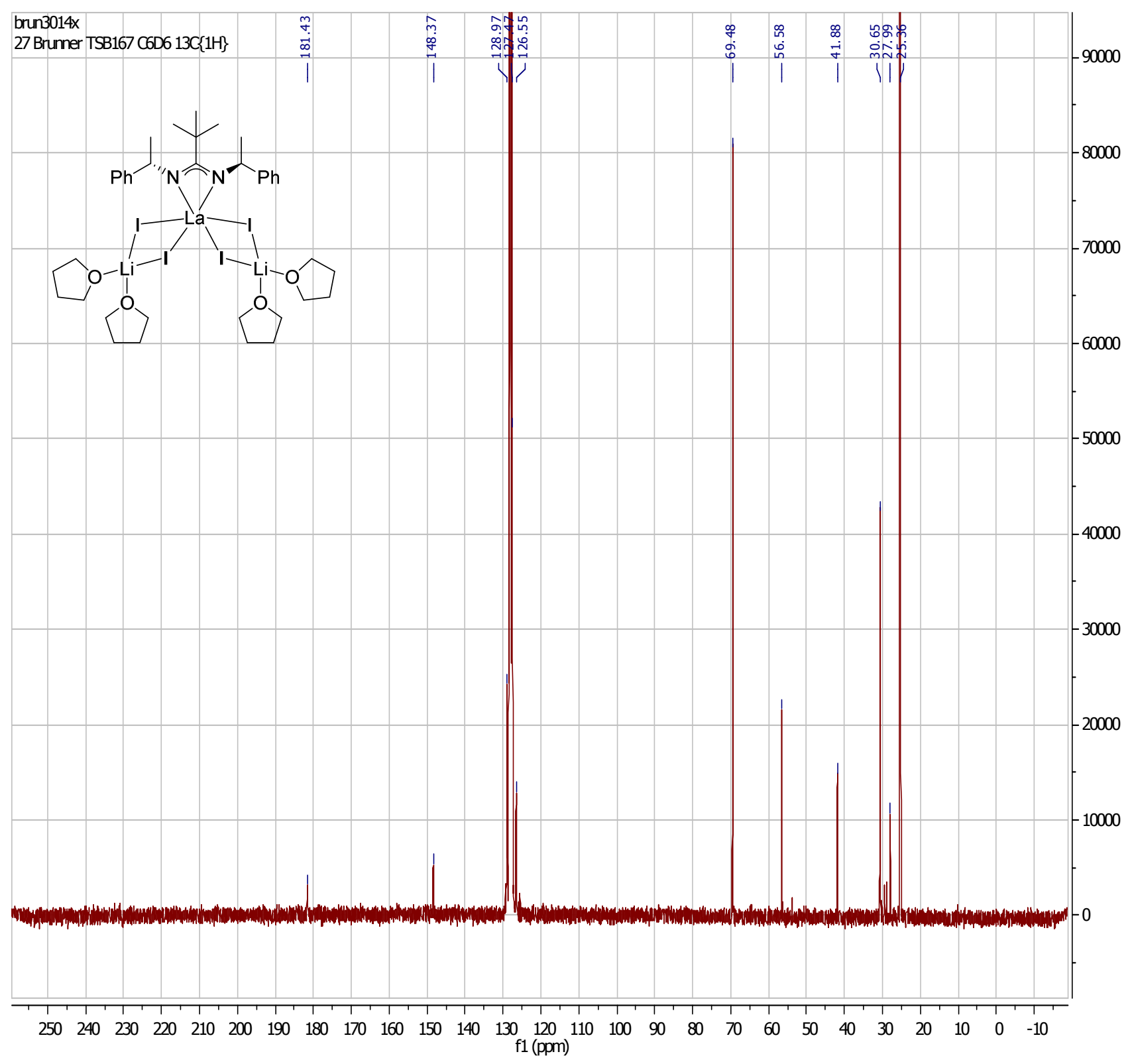


Figure S20. ${ }^{1} \mathrm{H}$ NMR of $2\left(300 \mathrm{MHz}, \mathrm{C}_{6} \mathrm{D}_{6}, 298 \mathrm{~K}\right)$.

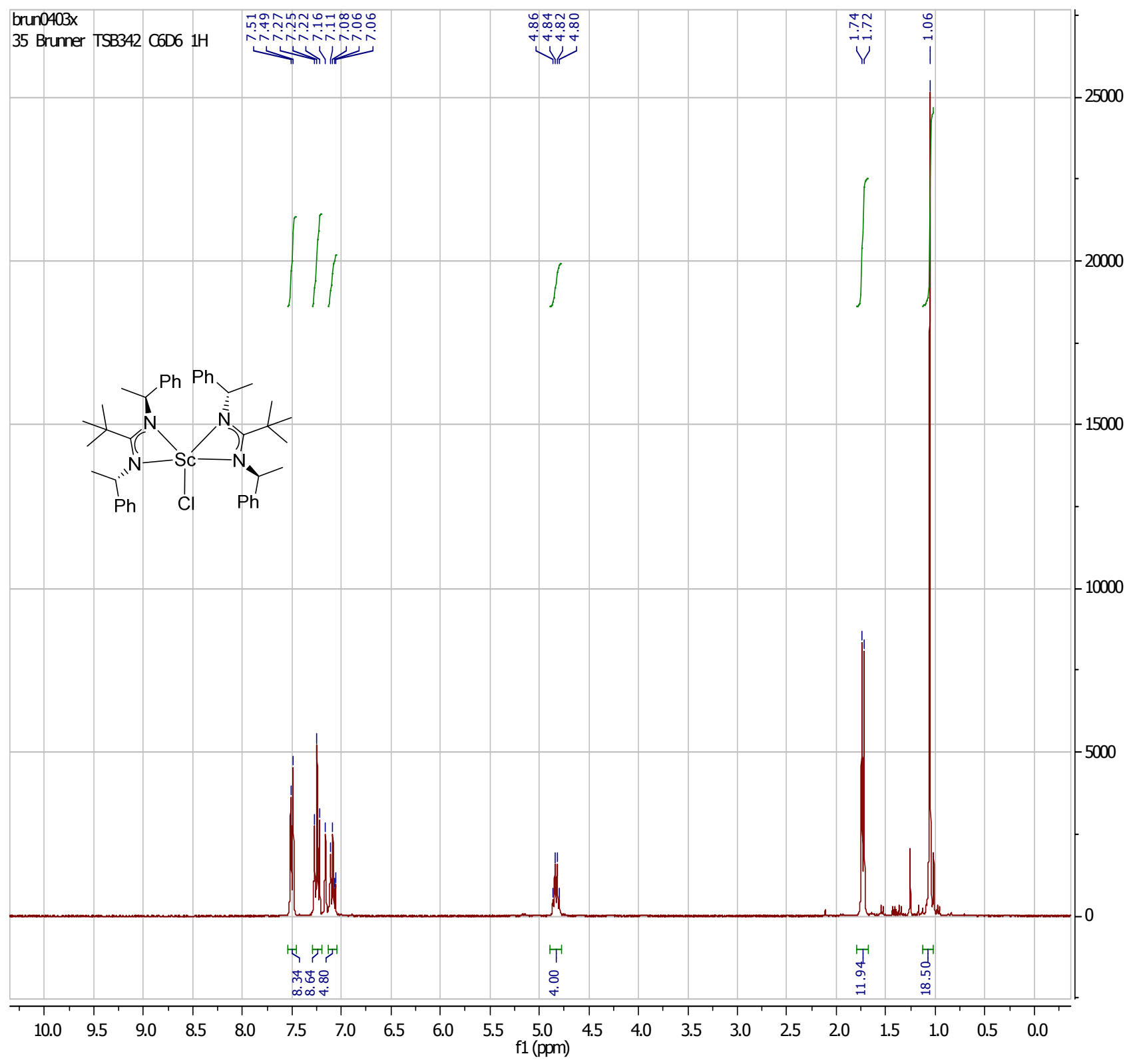


Figure S21. ${ }^{13} \mathrm{C}\left\{{ }^{1} \mathrm{H}\right\}$ NMR of $2\left(75 \mathrm{MHz}, \mathrm{C}_{6} \mathrm{D}_{6}, 298 \mathrm{~K}\right)$.

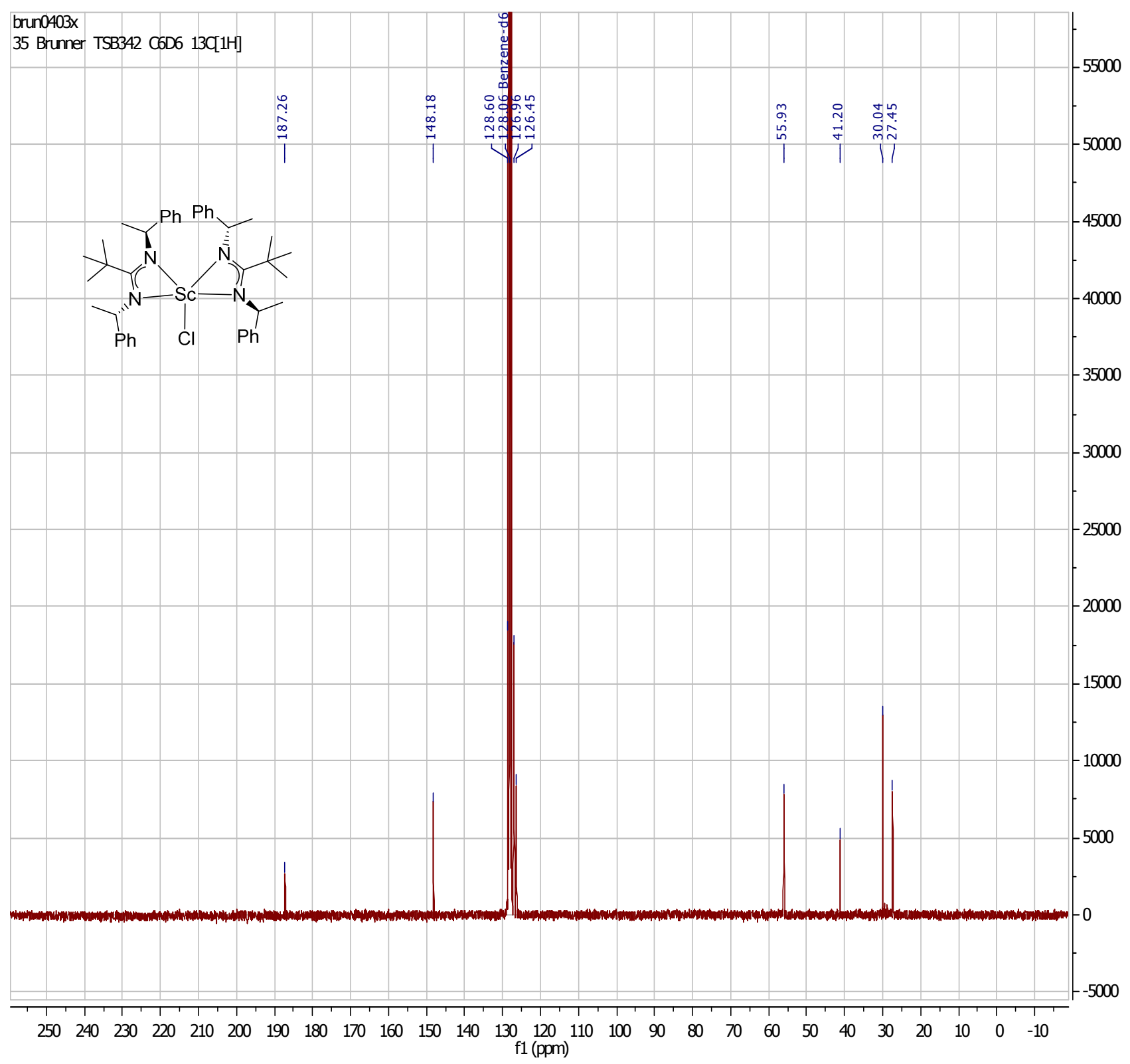


Figure S22. ${ }^{1} \mathrm{H}$ NMR of $3\left(300 \mathrm{MHz}, \mathrm{C}_{6} \mathrm{D}_{6}, 298 \mathrm{~K}\right)$.

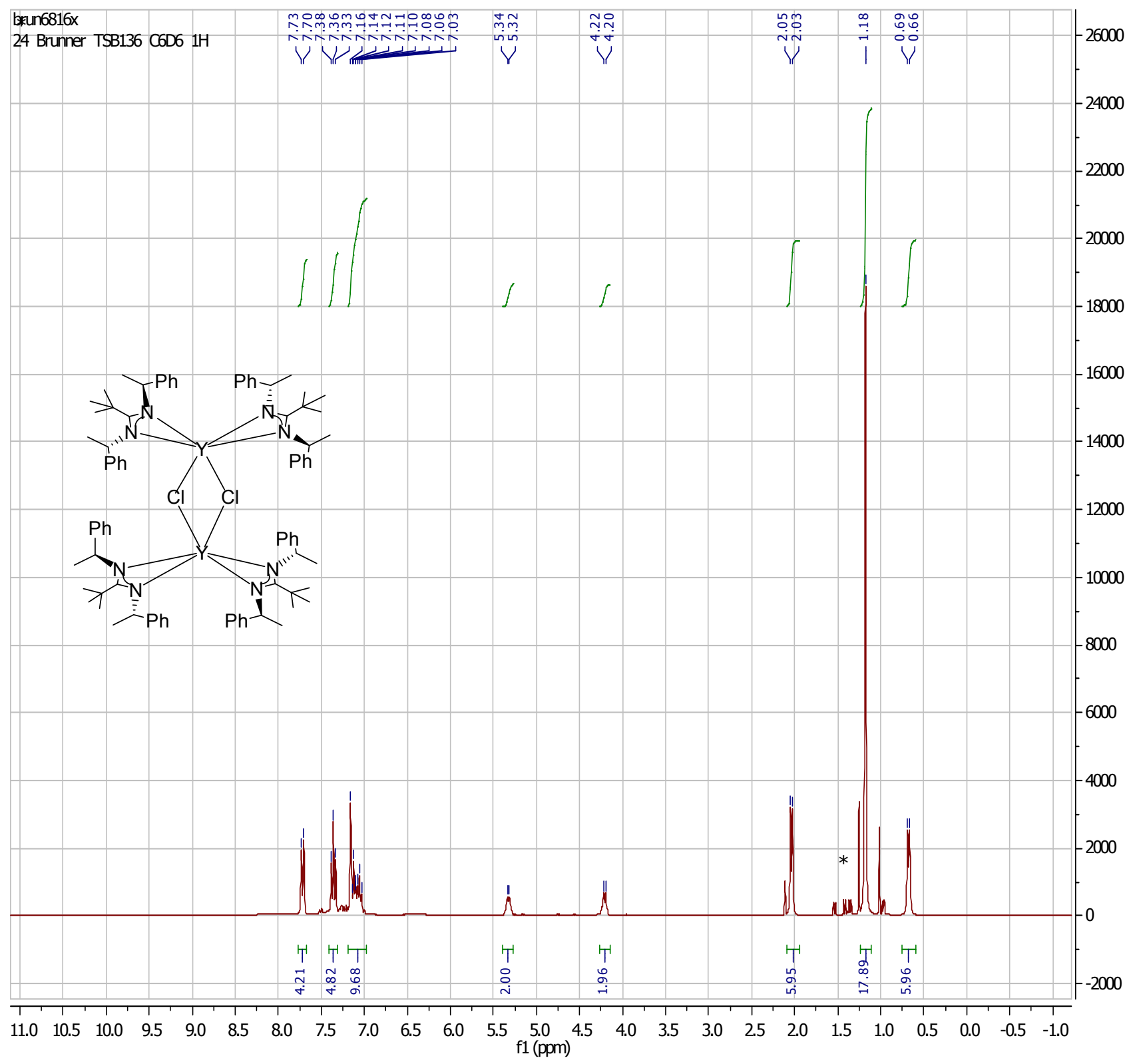

* minor impurities 
Figure S23. ${ }^{13} \mathrm{C}\left\{{ }^{1} \mathrm{H}\right\}$ NMR of $\mathbf{3}\left(75 \mathrm{MHz}, \mathrm{C}_{6} \mathrm{D}_{6}, 298 \mathrm{~K}\right)$.




Figure S24. ${ }^{1} \mathrm{H}$ NMR of 5 (300 MHz, D8-THF, $332 \mathrm{~K}$ ).

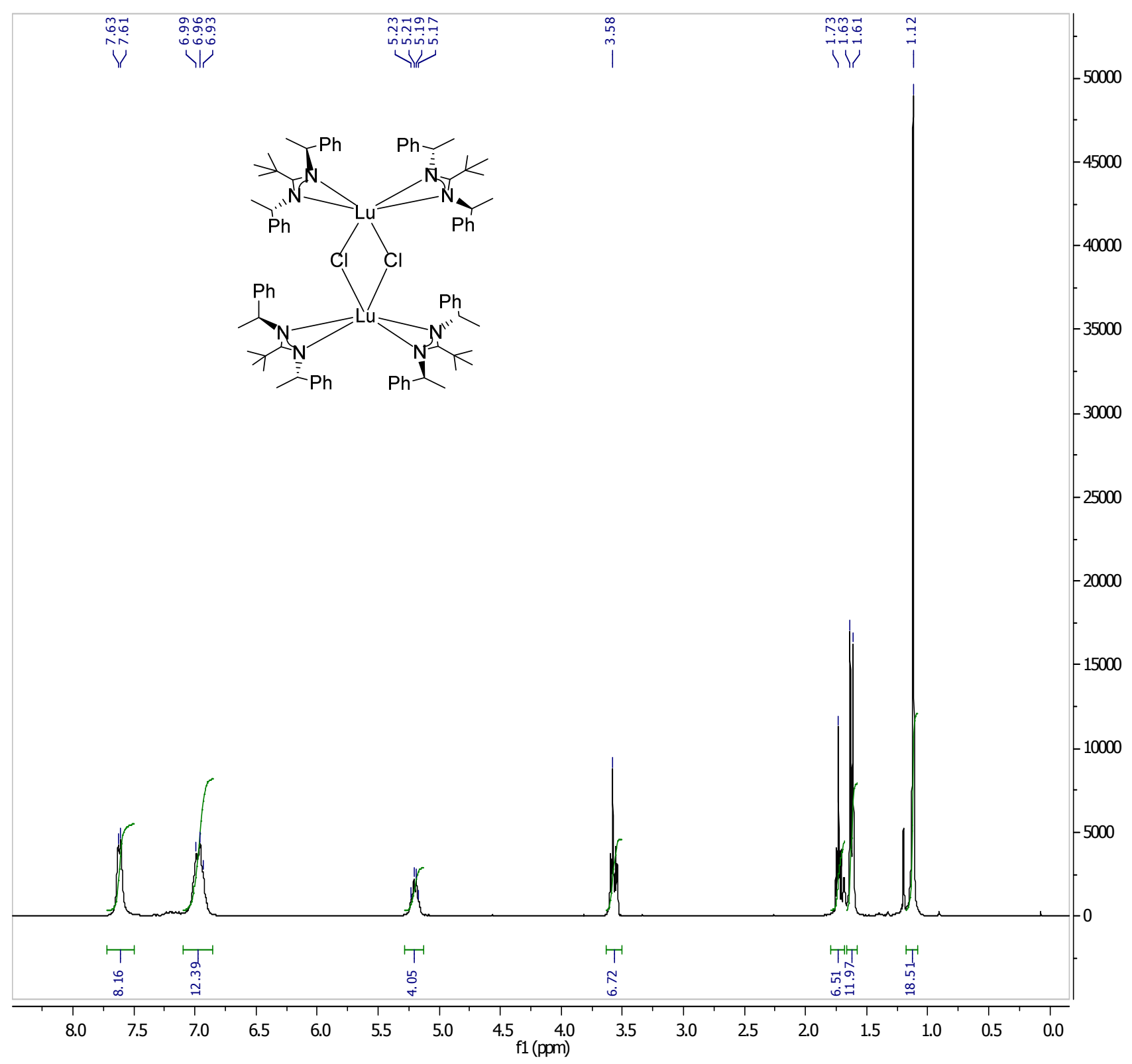


Figure S25. ${ }^{13} \mathrm{C}\left\{{ }^{1} \mathrm{H}\right\}$ NMR of $5(75 \mathrm{MHz}, \mathrm{D} 8-\mathrm{THF}, 332 \mathrm{~K})$.

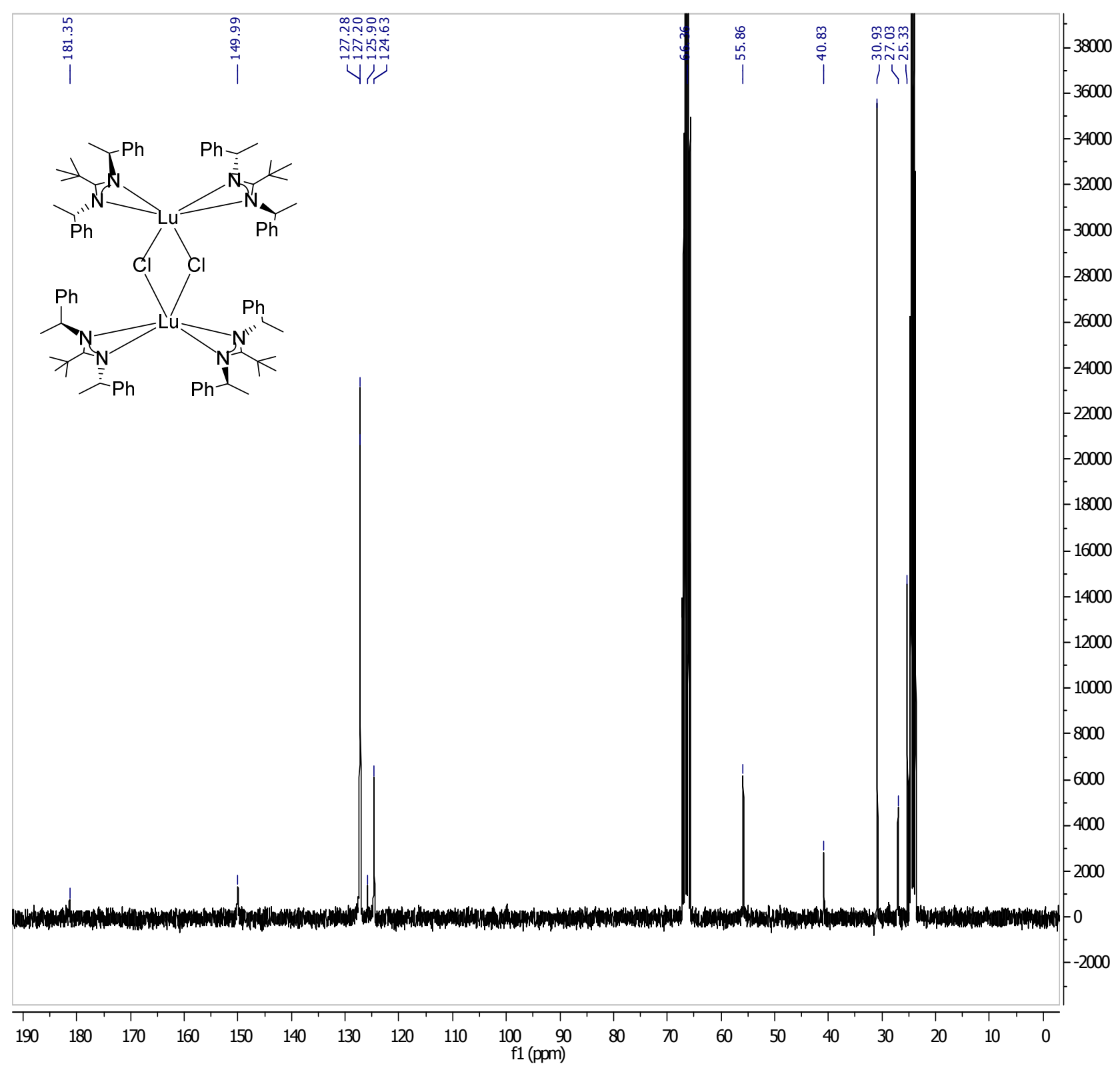


Figure S26. ${ }^{1} \mathrm{H}$ NMR of $7\left(400 \mathrm{MHz}, \mathrm{C}_{6} \mathrm{D}_{6}, 298 \mathrm{~K}\right)$.

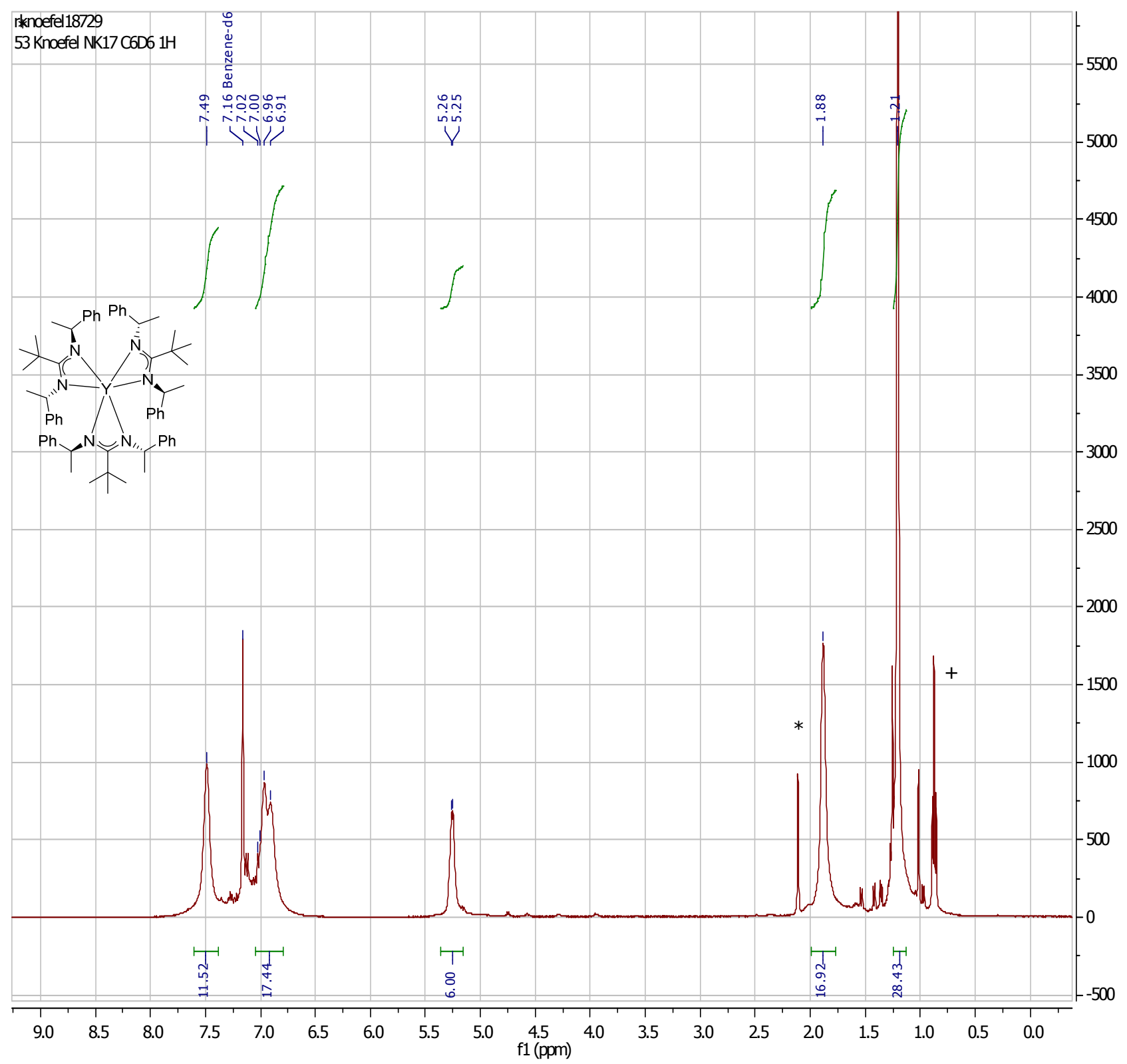

Minor impurities from toluene $\left({ }^{*}\right)$ and pentane $(+)$ 
Figure S27. ${ }^{13} \mathrm{C}\left\{{ }^{1} \mathrm{H}\right\}$ NMR of $7\left(101 \mathrm{MHz}, \mathrm{C}_{6} \mathrm{D}_{6}, 298 \mathrm{~K}\right)$.

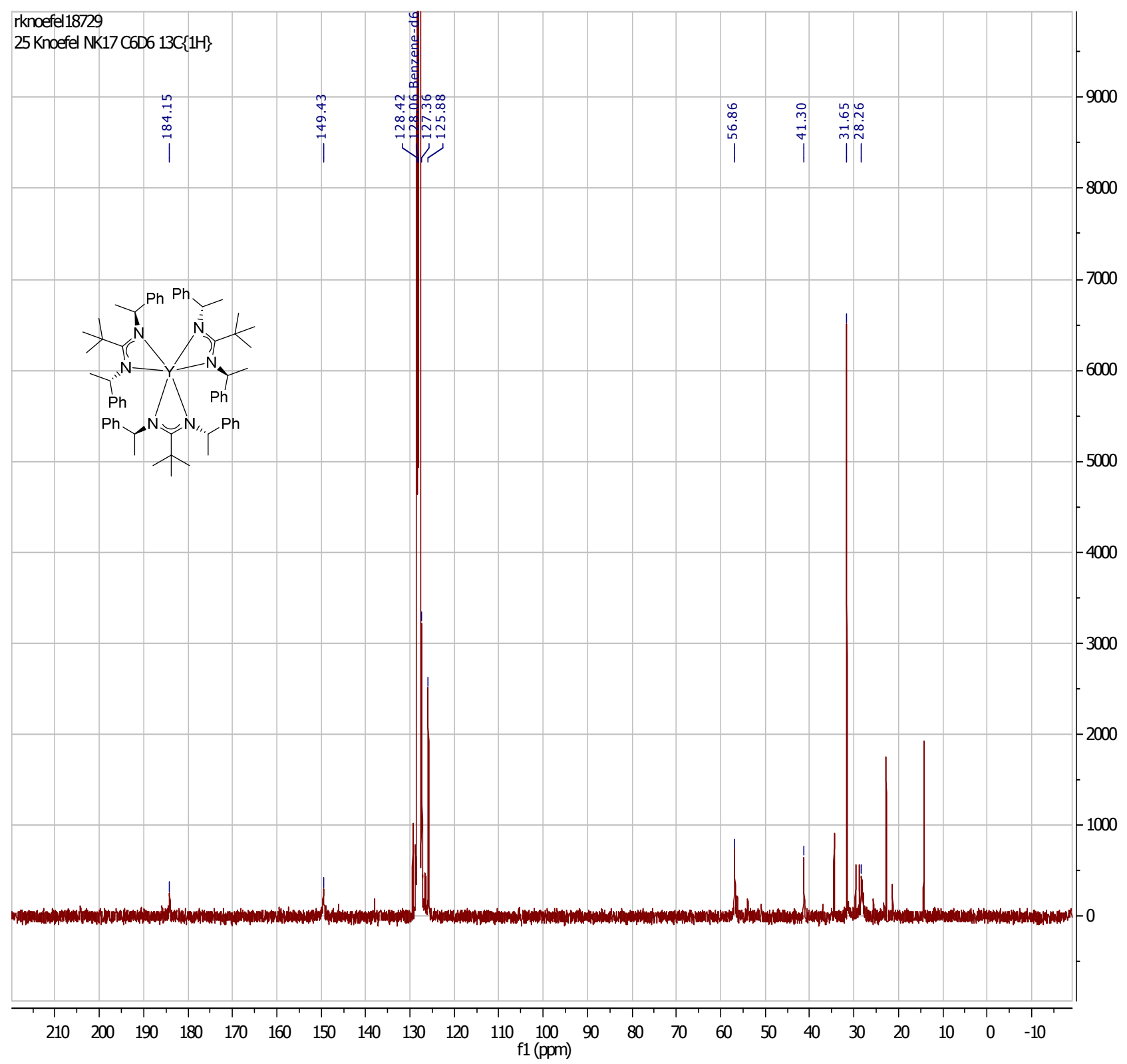

Minor impurities from toluene (38 und $129.34 \mathrm{ppm}$ und $34.4 \mathrm{ppm}$ ) and pentane (34.44 und 22.73 und $14.28 \mathrm{ppm}$ ) 
Figure S28. ${ }^{1} \mathrm{H}$ NMR of $8\left(400 \mathrm{MHz}, \mathrm{C}_{6} \mathrm{D}_{6}, 298 \mathrm{~K}\right)$.

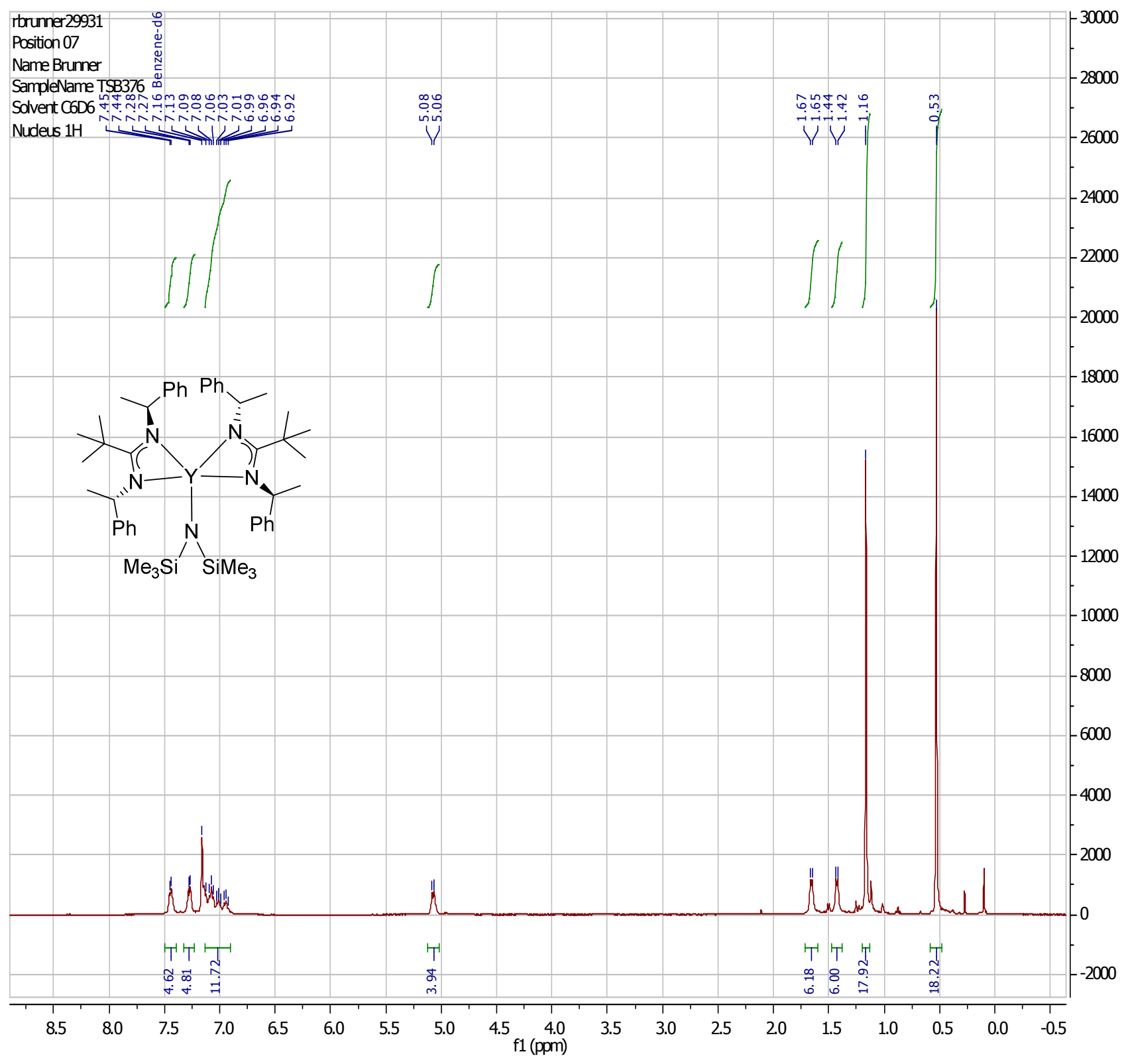


Figure S29. ${ }^{13} \mathrm{C}\left\{{ }^{1} \mathrm{H}\right\} \mathrm{NMR}$ of $8\left(101 \mathrm{MHz}, \mathrm{C}_{6} \mathrm{D}_{6}, 298 \mathrm{~K}\right)$.

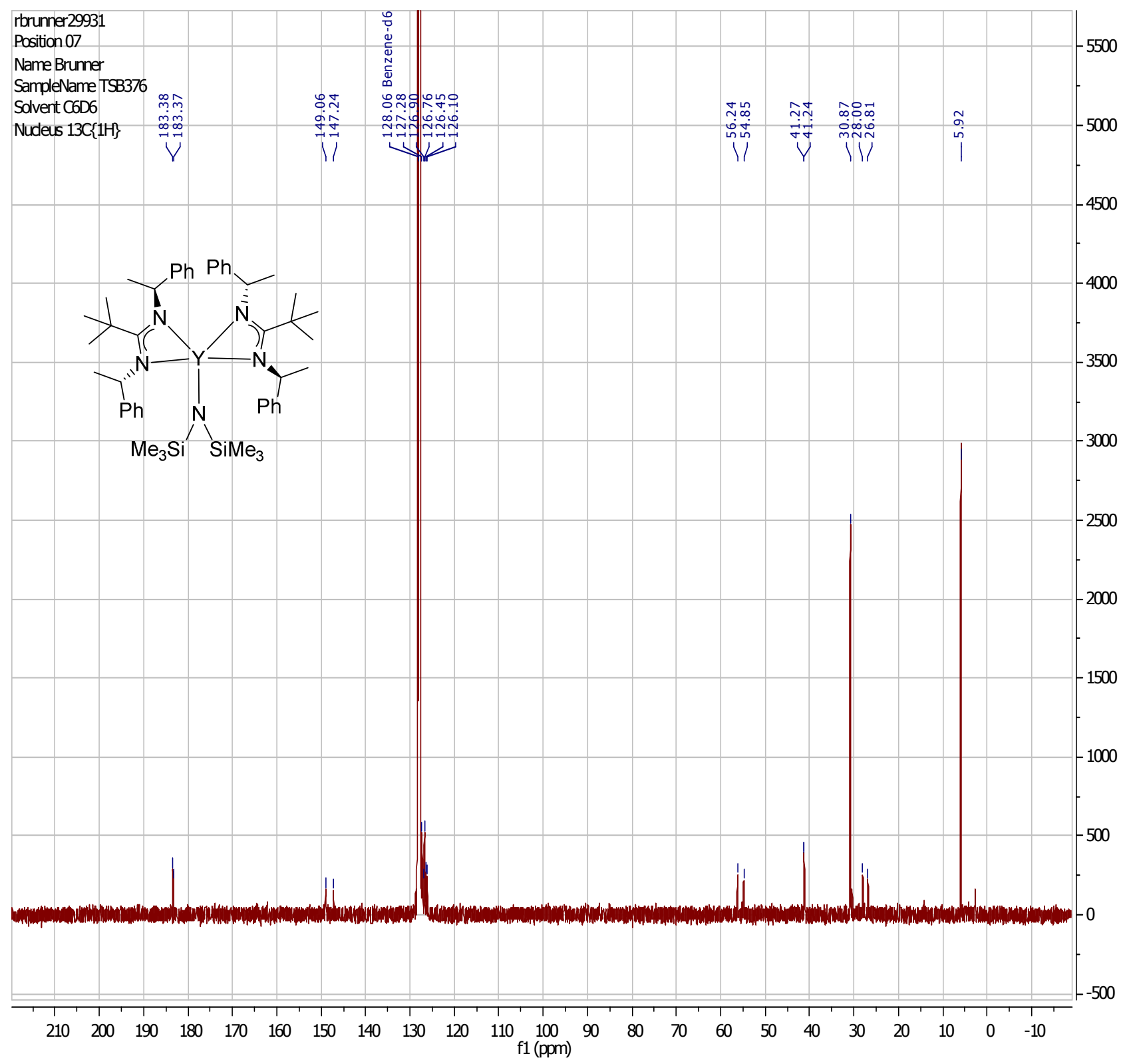


Figure S30. ${ }^{1} \mathrm{H}$ NMR of 9 (300 MHz, D8-THF, $\left.353 \mathrm{~K}\right)$.

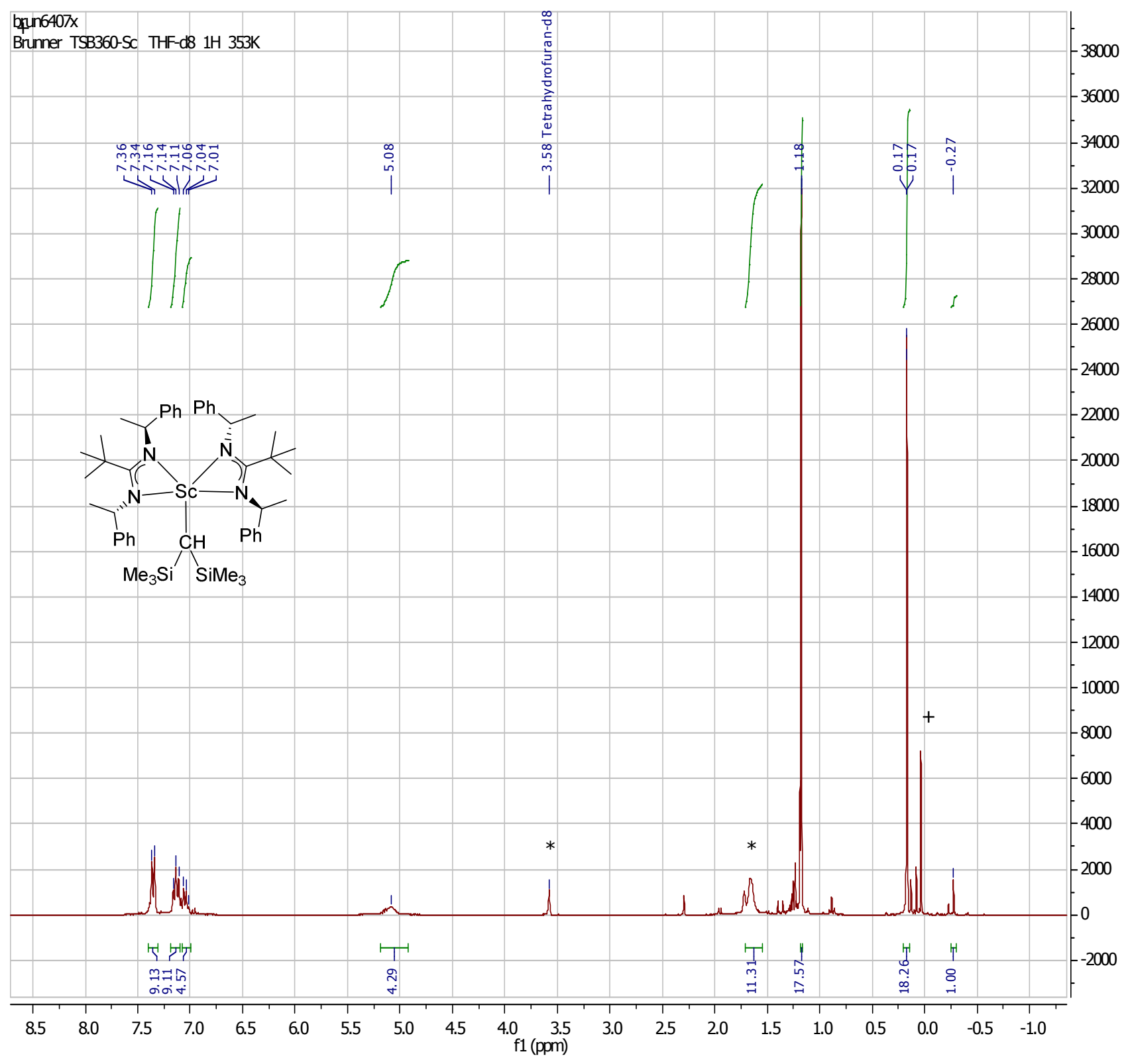

* = NMR solvent, $+\mathrm{CH}_{2} \mathrm{SiMe}_{3}$ 
Figure S31. ${ }^{13} \mathrm{C}\left\{{ }^{1} \mathrm{H}\right\}$ NMR of $9(75 \mathrm{MHz}, \mathrm{D} 8-\mathrm{THF}, 353 \mathrm{~K})$.

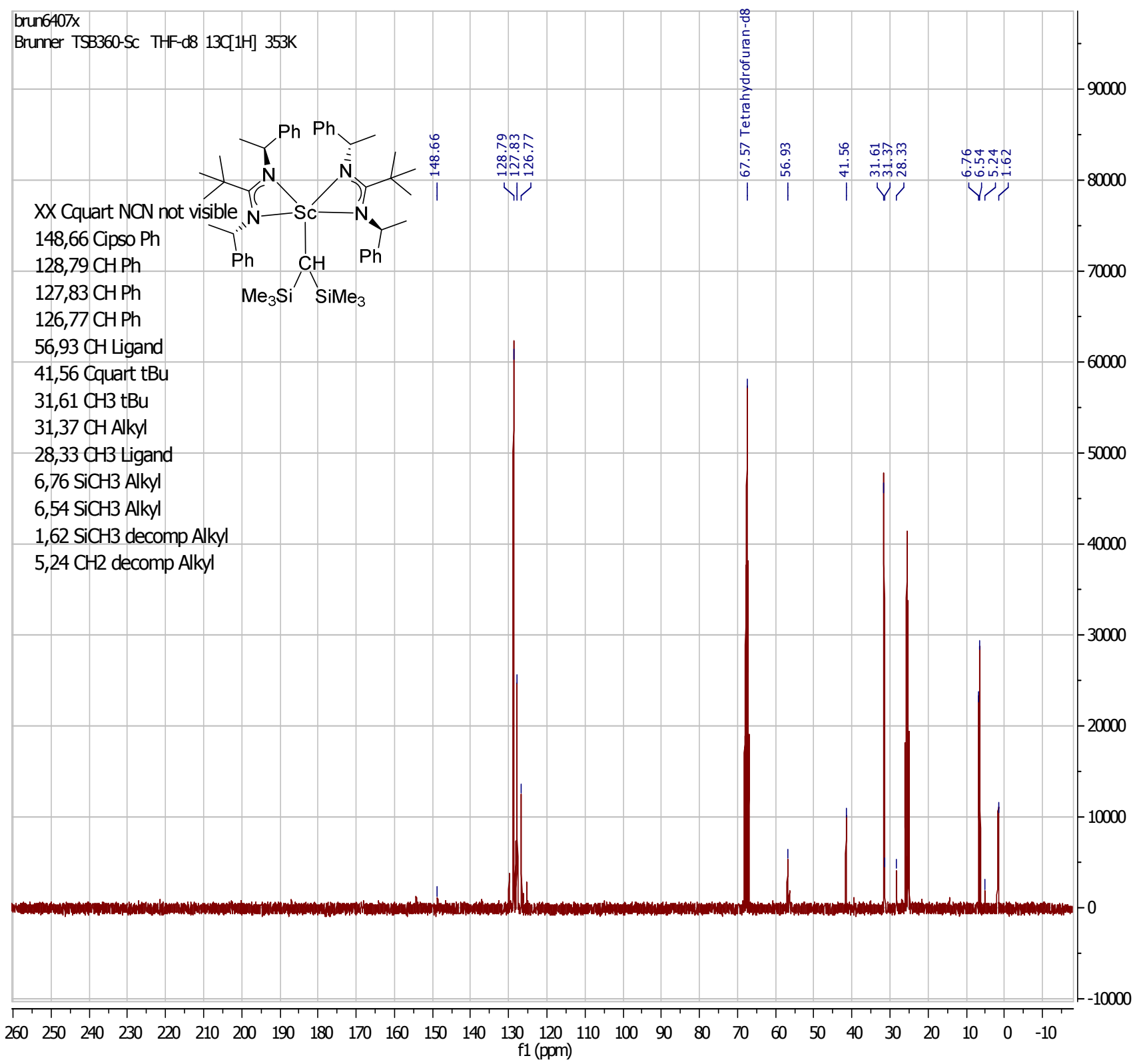


Figure S32. ${ }^{1} \mathrm{H}$ NMR of $\mathbf{1 0}(300 \mathrm{MHz}$, D8-THF, $353 \mathrm{~K}$ ).

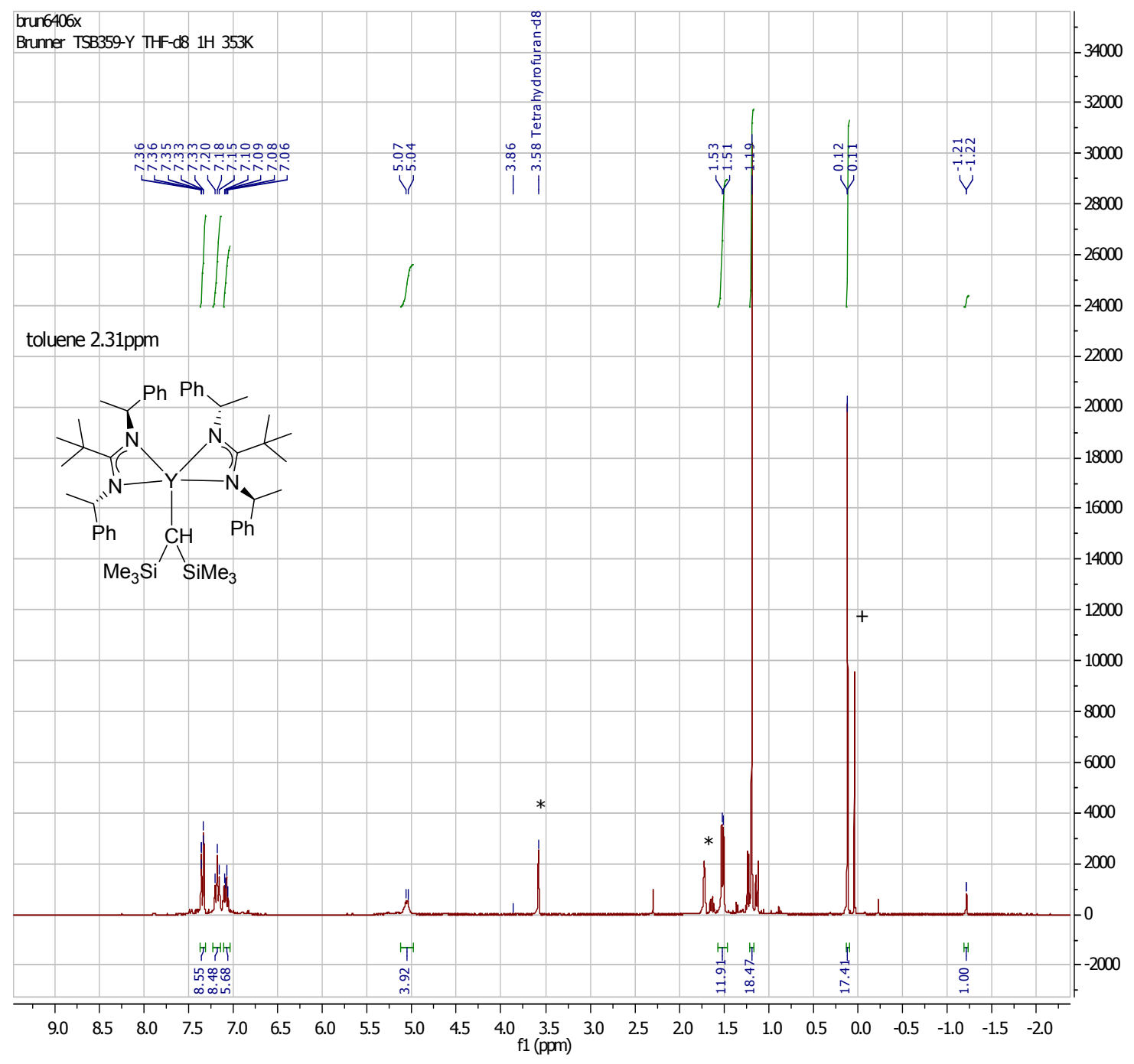

$*$ = NMR solvent, $+\mathrm{CH}_{2} \mathrm{SiMe}_{3}$ 
Figure S33. ${ }^{13} \mathrm{C}\left\{{ }^{1} \mathrm{H}\right\}$ NMR of $\mathbf{1 0}(75 \mathrm{MHz}$, D8-THF, $353 \mathrm{~K})$.

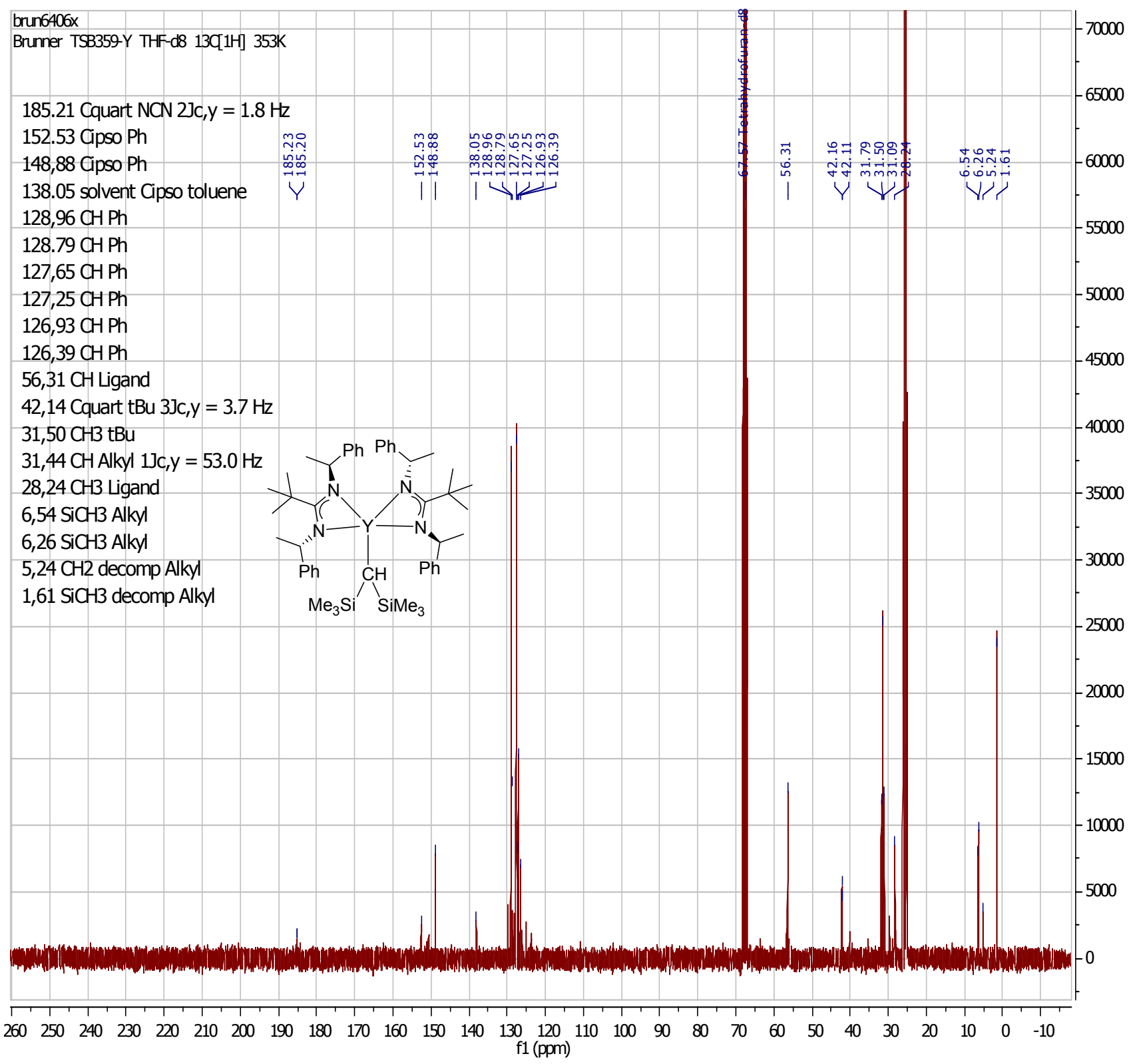


Figure S34. ${ }^{1} \mathrm{H}$ NMR of 11 (300 MHz, D8-THF, $353 \mathrm{~K}$ ).

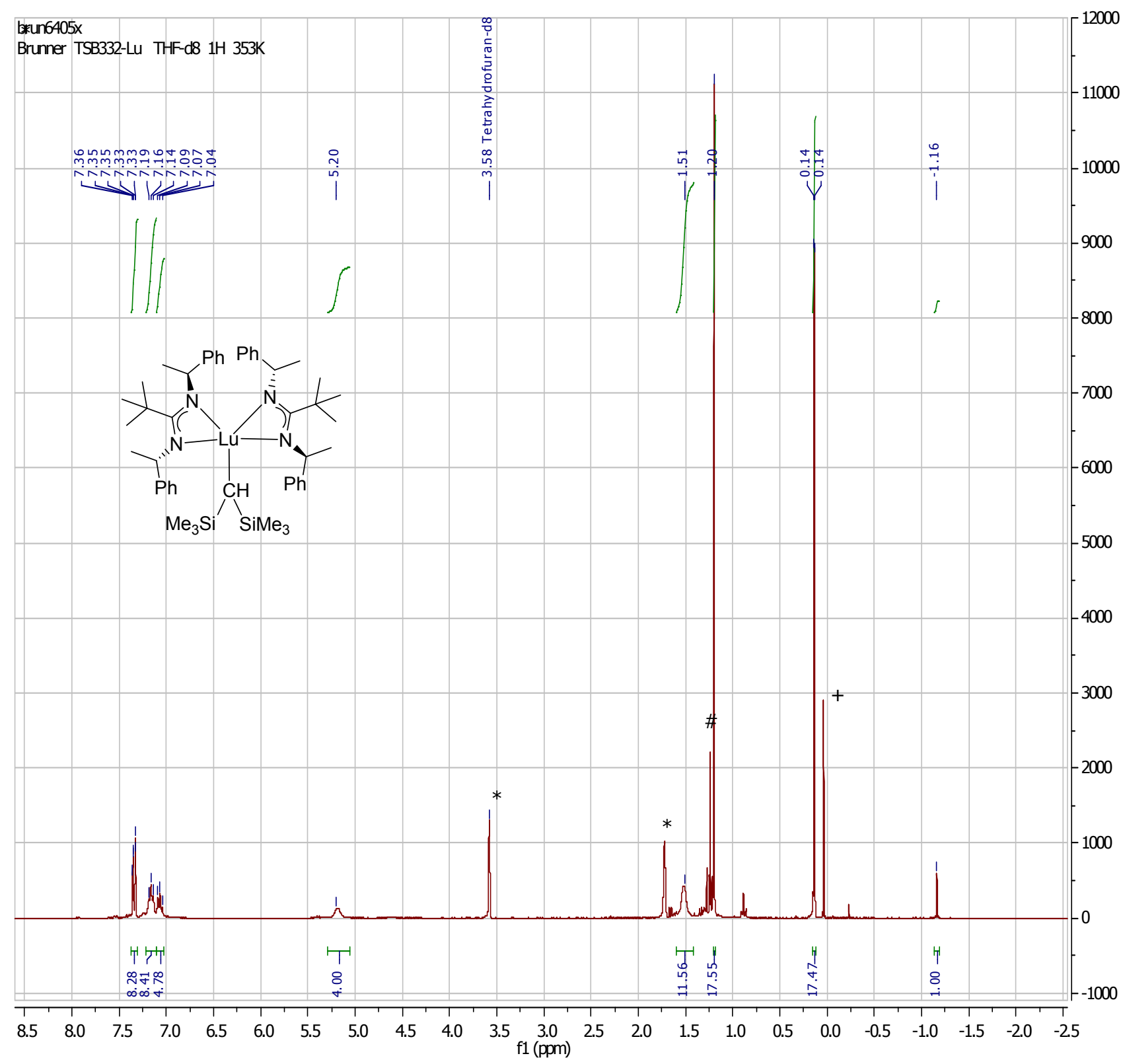

* = NMR solvent, $+\mathrm{CH}_{2} \mathrm{SiMe}_{3}$, \# ligand 
Figure S35. ${ }^{13} \mathrm{C}\left\{{ }^{1} \mathrm{H}\right\}$ NMR of $11(75 \mathrm{MHz}$, D8-THF, $353 \mathrm{~K})$.

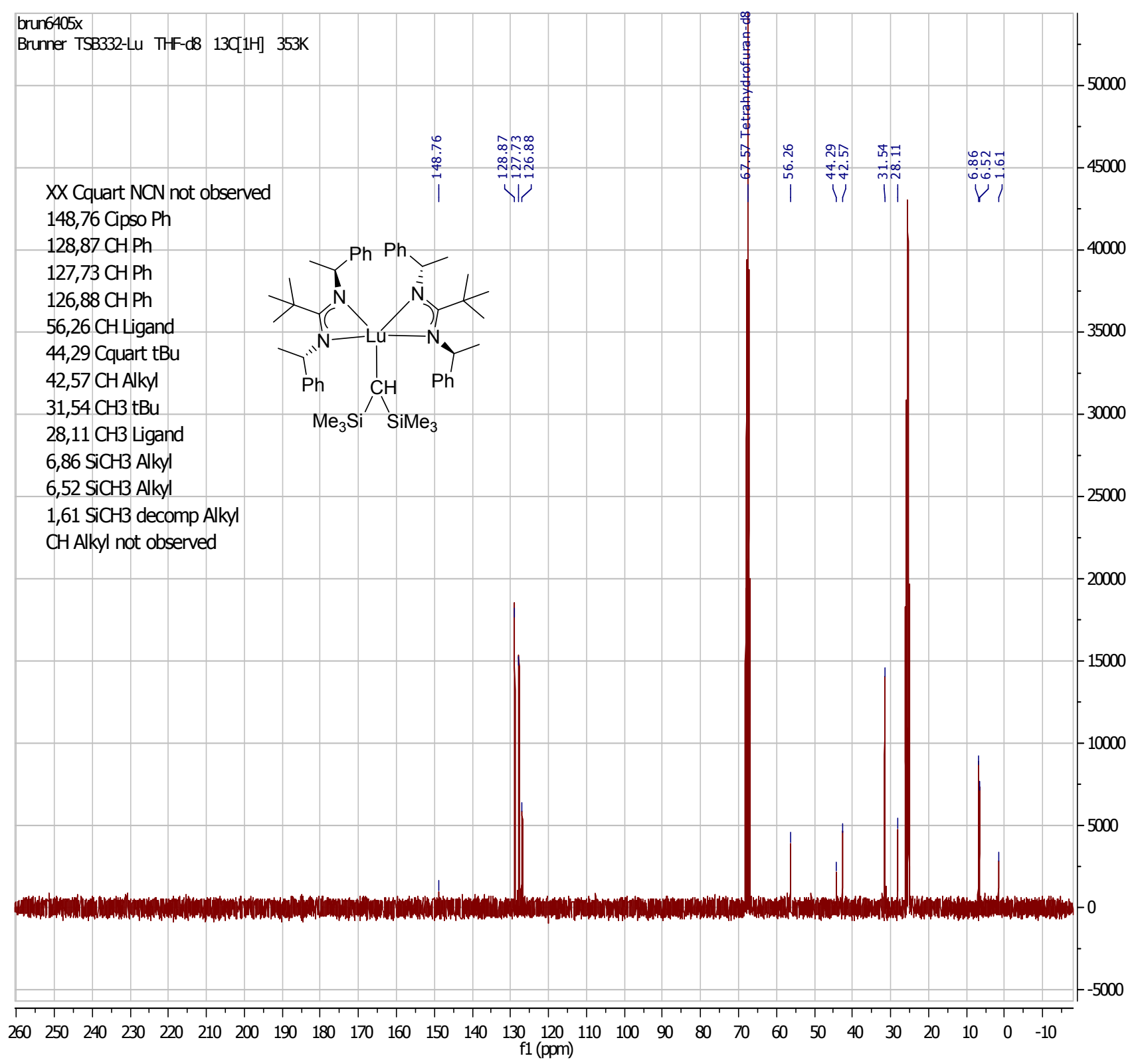


IR

Figure S36. IR Spectrum of (S)-1-Phenylethyl)carbodiimide ((S)-PEC).



\begin{tabular}{|c|c|c|c|}
\hline Samole Name & PB335 & Aperture Setting & $6 \mathrm{~mm}$ \\
\hline Sample Form & leicht oelbes Ö & Beamsolitter Selting & $\mathrm{KBr}$ \\
\hline Oderator Name & Default & Detector Settine & RT-DLaTGS Internal \\
\hline Resolution & 4 & Source Setting & MIR \\
\hline Scantime or Scans & & Measurement Channel & Samole Comoartment. \\
\hline Samole Scans & 64 & Acquisition Mode & Double Sided.Forward-Backward \\
\hline Start Frequency Limit for File & 4000 & Phase Correction Mode & Mertz \\
\hline End Frequency Limit for File & 500 & Apodization Function & Blackman-Harris 3-Term. \\
\hline
\end{tabular}

Seite 1 von 2 
Figure S37. IR Spectrum of lithium-N,N'-bis-((S)-1-phenylethyl)pivalamidinate ((S)-LiPETA).

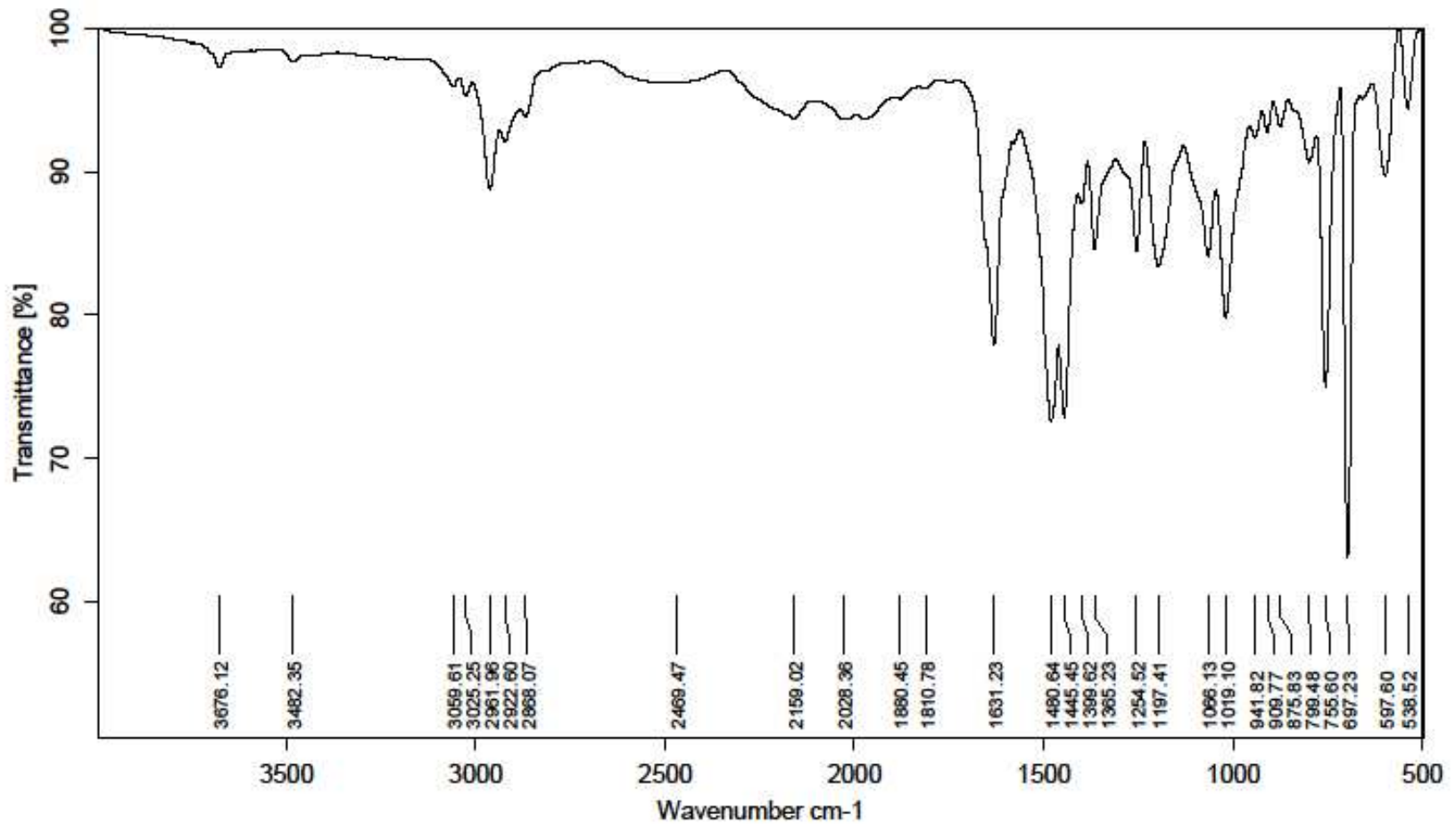

\begin{tabular}{|c|c|c|c|}
\hline Samole Name & PB335 & Amerture Setting & $6 \mathrm{~mm}$ \\
\hline Sample Form & oranoe rotes Pulver & Beamsolitter Selting & $\mathrm{KBr}$ \\
\hline Oderator Name & Default & Detector Settine & RT-DLaTGS Interna! \\
\hline Resolution & 4 & Source Selting & MIR \\
\hline Scantime or Scans & & Measurement Channel & Samole Comoartment. \\
\hline Samole Scans & 64 & Acquisition Mode & Double Sided.Fonward-Backward \\
\hline Start Frequency Limit for File & 4000 & Phase Correction Mode & Mertz \\
\hline End Frequency Limit for File & 500 & Acodization Function & Blackman-Harris 3-Term \\
\hline
\end{tabular}

Seite 1 von 2 
Figure S38. IR Spectrum of $\left[\{(S)-\mathrm{PETA}\} \mathrm{La}(\mu-\mathrm{I})_{4} \mathrm{Li}_{2}(\text { thf })_{4}\right](\mathbf{1})$.

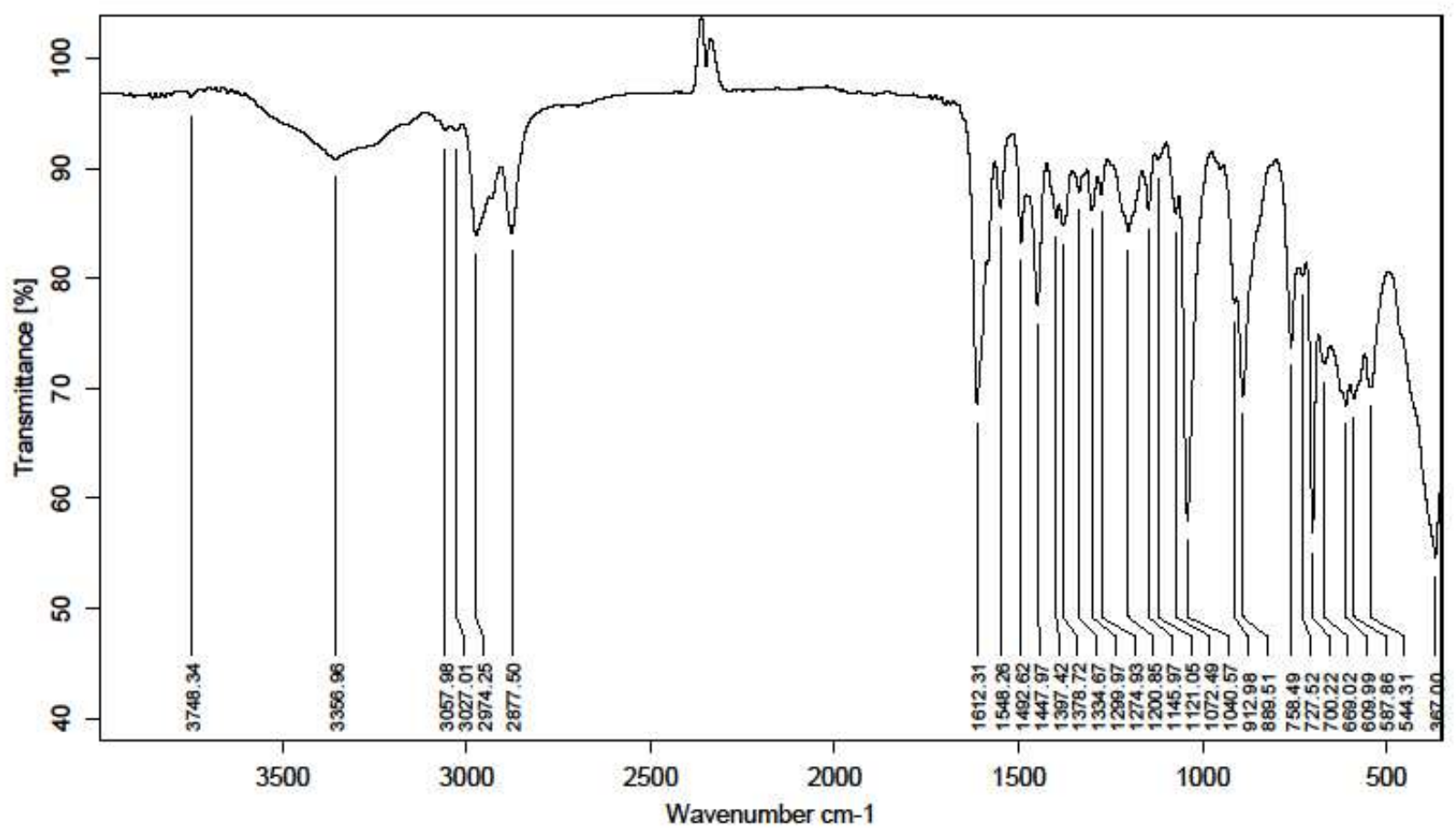

\begin{tabular}{|c|c|c|c|}
\hline Samole Name & (PEIA)Lal2 nochmal & Aderture Setting & $6 \mathrm{~mm}$ \\
\hline Sample Form & Pulver & Beamsolitter Selting & $\mathrm{KBr}$ \\
\hline Oderator Name & Default: & Detector Settina & RT-DLaTGS Internal \\
\hline Resolution & 4 & Source Setting & MIR \\
\hline Scantime or Scans & & Measurement Channel & Samole Comoartment \\
\hline Samole Scans & 64 & Acquisition Mode & Double Sided.Forward-Backward \\
\hline Start Freouency Limit for File & 4000 & Phase Correction Mode & Mertz \\
\hline End Frequencr Limit for File & 350 & Anodization Function & Blackman-Harris 3-Term. \\
\hline
\end{tabular}

Seite 1 von 2 
Figure S39. IR Spectrum of $\left[\{(S)-\mathrm{PETA}\}_{2} \mathrm{ScCl}\right](2)$.

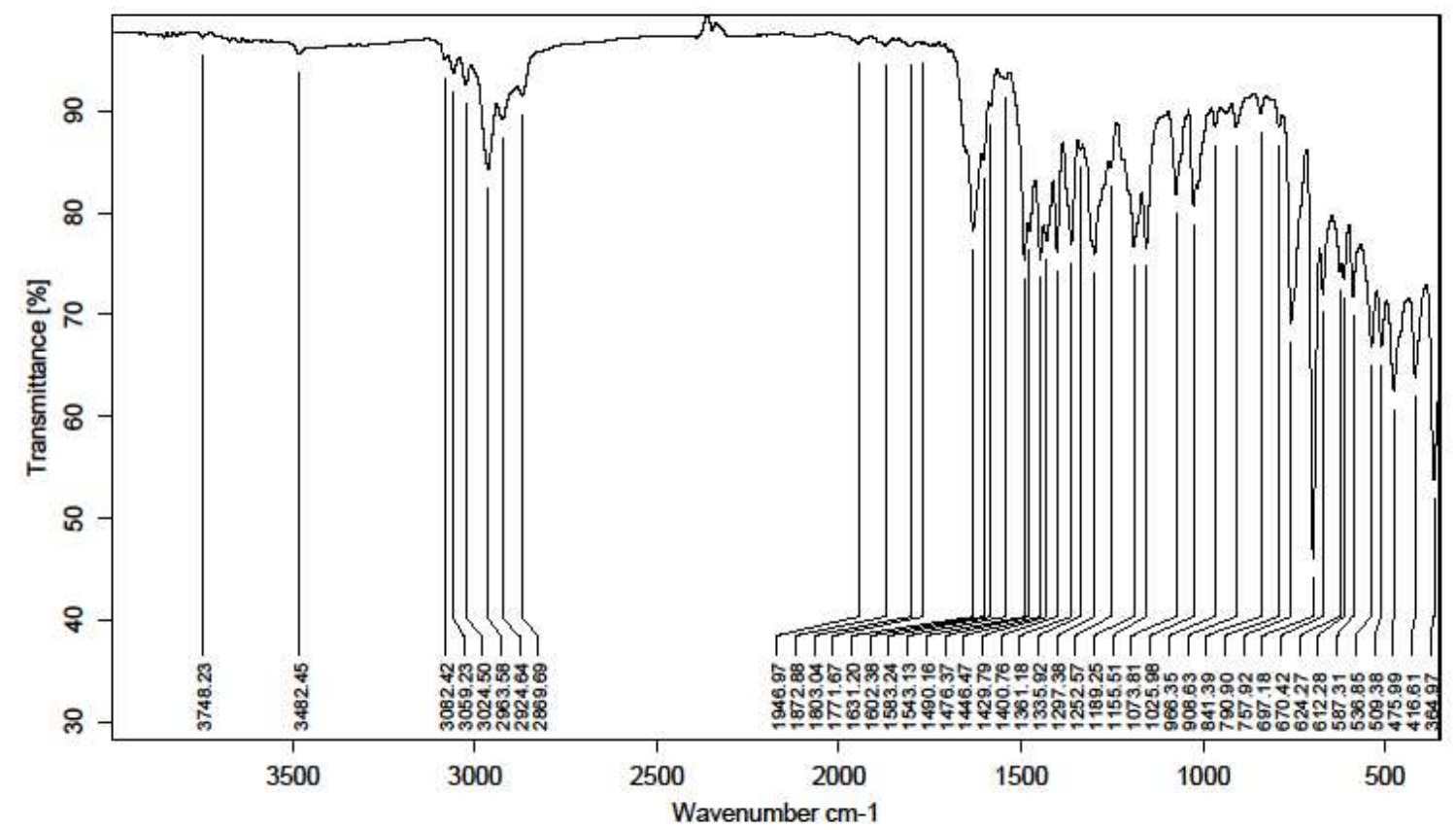

\begin{tabular}{|c|c|c|c|}
\hline Samole Name & (PETA)2SCCl & Averture Setting & $6 \mathrm{~mm}$ \\
\hline Samole Form & Pulver & Beamsolitter Setting & $\mathrm{KBr}$ \\
\hline Operator Name & Default: & Detector Setting & RT-DLaTGS Internall \\
\hline Resolution & 4 & Source Selting & MIR \\
\hline Scantime or Scans & & Measurement Channel & Samole Compartment \\
\hline Samole Scans & 64 & Acavisition Made & Double Sided.Forward-Backward \\
\hline Start Frequency L imit for File & 4000 & Phase Correction Mode & Mertz \\
\hline End Freouencr Limit for File & 350 & Anodization Function & Blackman-Harris 3-Term \\
\hline
\end{tabular}

Seite 1 von 2 
Figure S40. IR Spectrum of $\left[\{(S)-\mathrm{PETA}\}_{2} \mathrm{YCl}\right]_{2}(\mathbf{3})$.

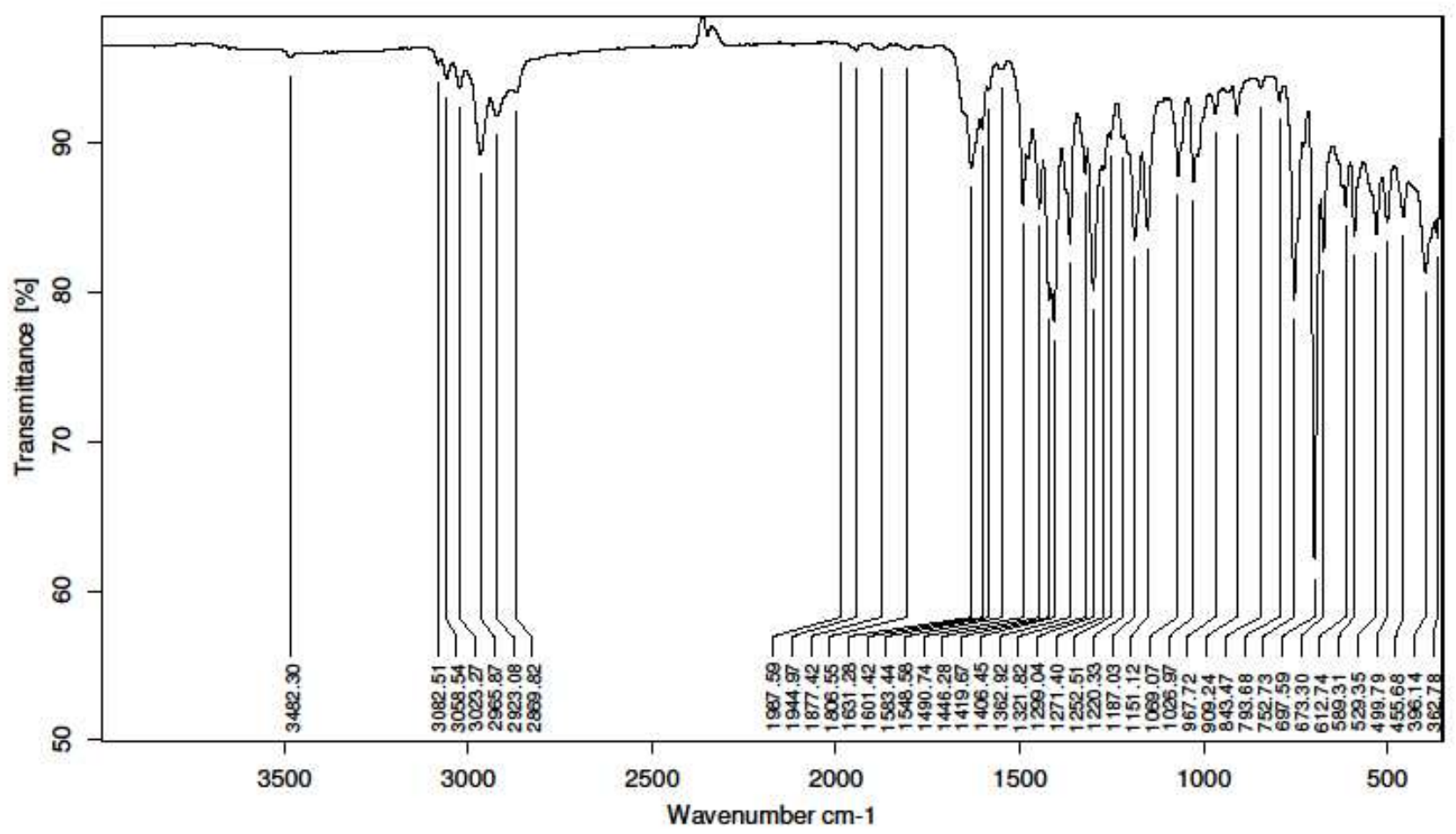

\begin{tabular}{|c|c|c|c|}
\hline Sample Name & (PETA)2YCL & Aperture Selting & $6 \mathrm{~mm}$ \\
\hline Samole Form & Pulver & Beamsolitter Selting & $\mathrm{KBr}$ \\
\hline Ooerator Name & Default & Dotecter Setting & RT-DLaTGS flnternall \\
\hline Aesolution & 4 & Source Setting & MiR \\
\hline Scantime or Scans & & Measurement Channel & Sample Comoartment \\
\hline Sample Scans & 64 & Acquisition Mode & Double Sidad.Forward-Backward \\
\hline Start Frequancy Limit for File & 4000 & Phase Correction Mods & Mertz \\
\hline End Frequency Limit for File & 350 & Apodization Function & Blackman-Harris 3-Term. \\
\hline
\end{tabular}

Seite 1 von 2 
Figure S41. IR Spectrum of $\left[\{(S)-P E T A\}_{2} \mathrm{NdCl}\right]_{2}(4)$.

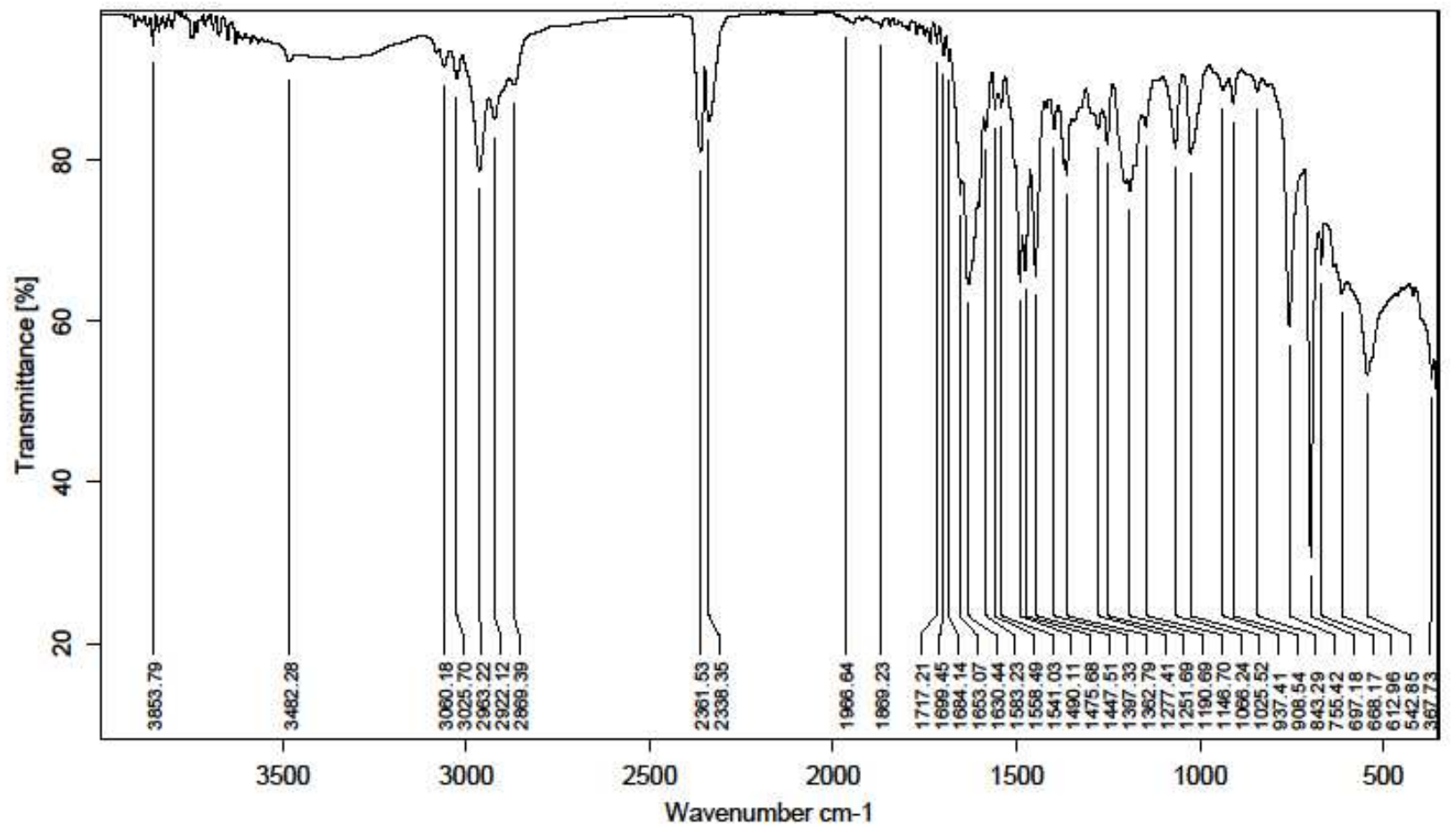

\begin{tabular}{|c|c|c|c|}
\hline Samole Name & (PETA) $2 \mathrm{NdCl}$ & Acerture Setting & $6 \mathrm{~mm}$ \\
\hline Sample Form & Pulver & Beamsolitter Selting & $\mathrm{KBr}$ \\
\hline Oderator Name & Default & Detector Setting & RT-DLaTGS Internaln \\
\hline Resolution & 4 & Source Setting & MIR \\
\hline Scantime or Scans & & Measurement Channel & Sample Comoartment \\
\hline Samole Scans & 64 & Acquisition Mode & Double Sided.Forward-Backward \\
\hline Start Frequency Limit for File & 4000 & Phase Correction Mode & Mertz \\
\hline End Frequency Limit for File & 350 & Acodization Function & Blackman-Harris 3-Term \\
\hline
\end{tabular}

Seite 1 von 2 
Figure S42. IR Spectrum of $\left[\{(S)-P E T A\}_{2} \mathrm{LuCl}\right]_{2}(\mathbf{5})$.

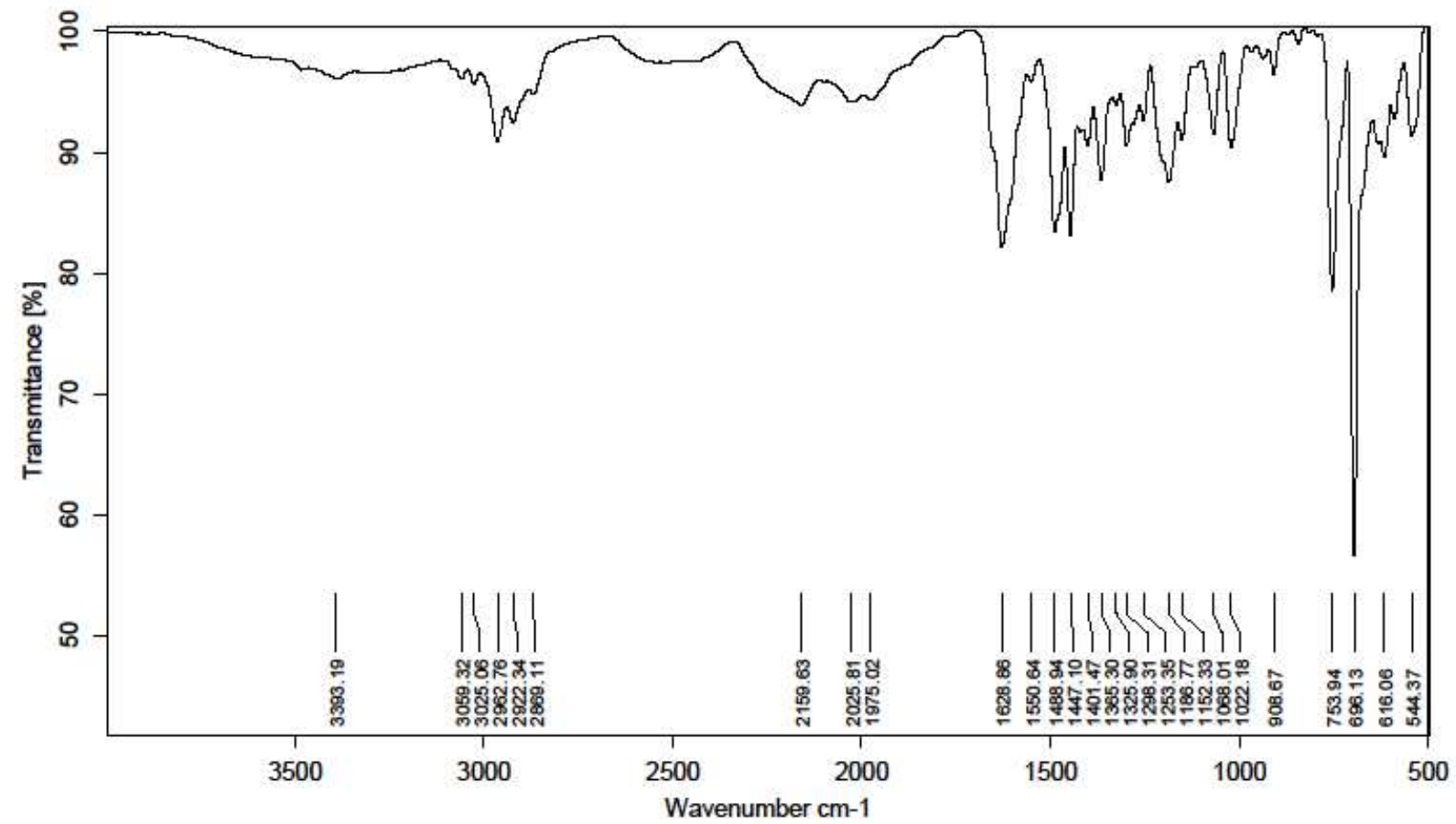

\begin{tabular}{|c|c|c|c|}
\hline Samole Name & TBOO & Acerture Setting & $6 \mathrm{~mm}$ \\
\hline Sample Form & oelbe Kristalle & Beamsolitter Selting & $\mathrm{KBr}$ \\
\hline Operator Name & Default & Detector Settine & RT-DLaTGS Internal] \\
\hline Resolution & 4 & Source Selting & MIR \\
\hline Scantime or Scans & & Measurement Channel & Samole Comoartment. \\
\hline Samole Scans & 64 & Acquisition Mode & Double Sided.Forward-Backward \\
\hline Start Frequency Limit for File & 4000 & Phase Correction Mode & Mertz \\
\hline End Frequency Limit for File & 500 & Anodization Function & Blackman-Harnis 3-Term \\
\hline
\end{tabular}

Seite 1 von 2 
Figure S43. IR Spectrum of $\left[\{(S)-\operatorname{PETA}\}_{2} \operatorname{Sm}(\mu-\mathrm{Cl})_{2} \operatorname{Li}(\text { thf })_{2}\right](6)$.

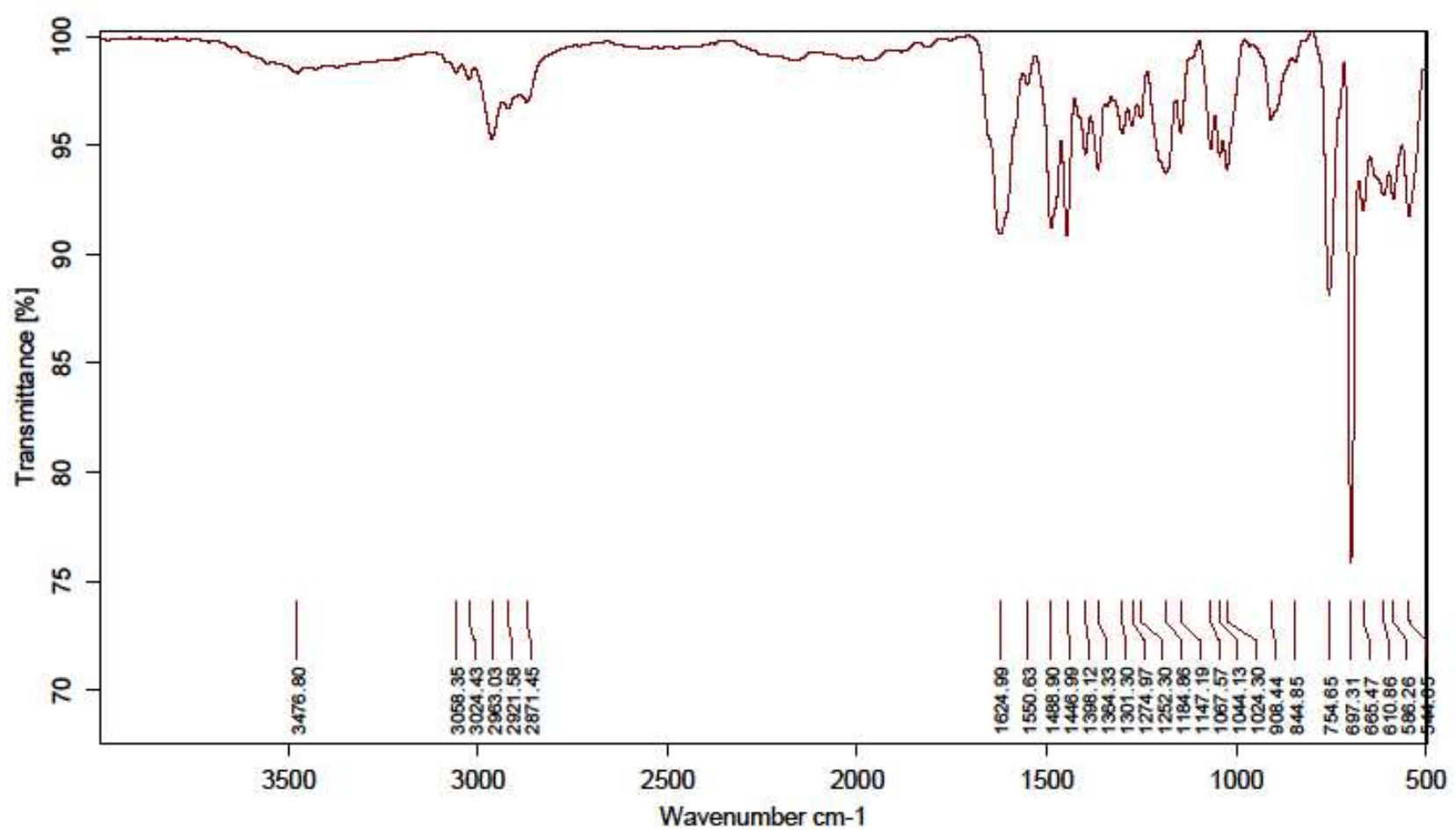

\begin{tabular}{|c|c|c|c|}
\hline Samole Name & PB350 & Averture Settinc & $6 \mathrm{~mm}$ \\
\hline Sample Form & oelbes Pulver & Beamsolitter Setting & $\mathrm{KBr}$ \\
\hline Oderator Name & Default & Detector Setting & RT-DLaTGS flnternall \\
\hline Resolution & 4 & Source Setting & MIR \\
\hline Scantime or Scans & & Measurement Channel & Sample Comvartment \\
\hline Sample Scans & 84 & Acquisition Made & Double Sided.Forwand-Backward \\
\hline Start Freouency L Limit for File & 4000 & Phase Correction Mode & Mertz \\
\hline End Frequency Limit for File & 500 & Aoodization Function & Blackman-Harris 3-Term \\
\hline
\end{tabular}

Seite 1 von 2 
Figure S44. IR Spectrum of $\left[\{(S)-\mathrm{PETA}\}_{2} \mathrm{Y}\left\{\mathrm{N}\left(\mathrm{SiMe}_{3}\right)_{2}\right\}\right]$ (8).

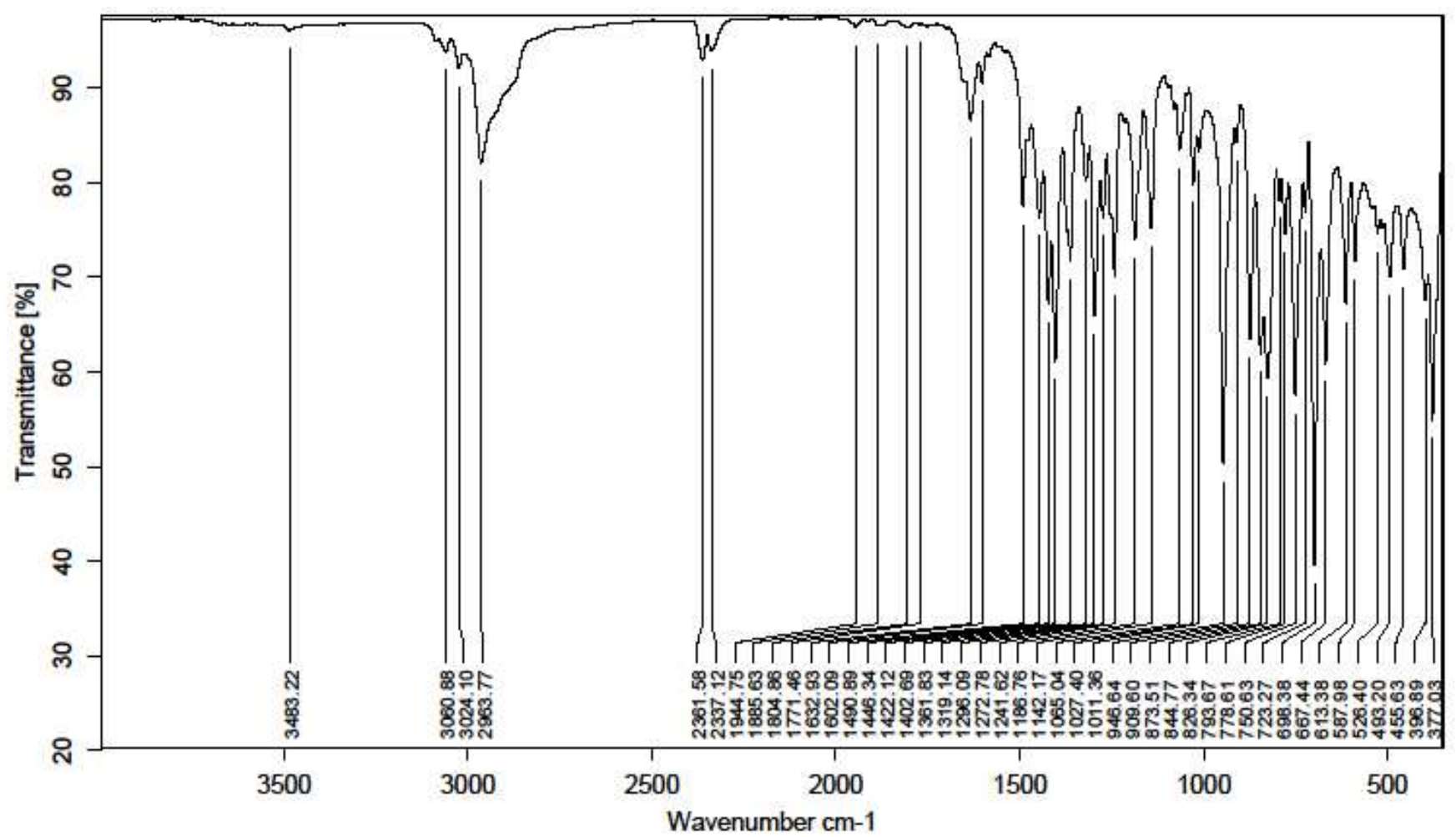

\begin{tabular}{|c|c|c|c|}
\hline Samole Name & (PETA)2Y(btsa) & Aperture Setting & $6 \mathrm{~mm}$ \\
\hline Sample Form & Pulver & Bearmsolitter Setting & $\mathrm{KBr}$ \\
\hline Operator Name & Default & Detector Setting & RT-DLaTGS Interna! \\
\hline Resolution & 4 & Source Selting & MIR \\
\hline Scantime or Scans & & Measurement Channel & Sample Comoartment. \\
\hline Samole Scans & 64 & Acquisition Mode & Double Sided.Forward-Backward \\
\hline Start Frequency Limit for File & 4000 & Phase Correction Made & Mertz \\
\hline Fnd Frequency Limit for File & 350 & Arodization Function & Blackman-Harris 3-Term \\
\hline
\end{tabular}

Seite 1 von 2 
Figure S45. IR Spectrum of $\left[\{(S)-\mathrm{PETA}\}_{2} \mathrm{Sc}\left\{\mathrm{CH}\left(\mathrm{SiMe}_{3}\right)_{2}\right\}\right]$ (9).

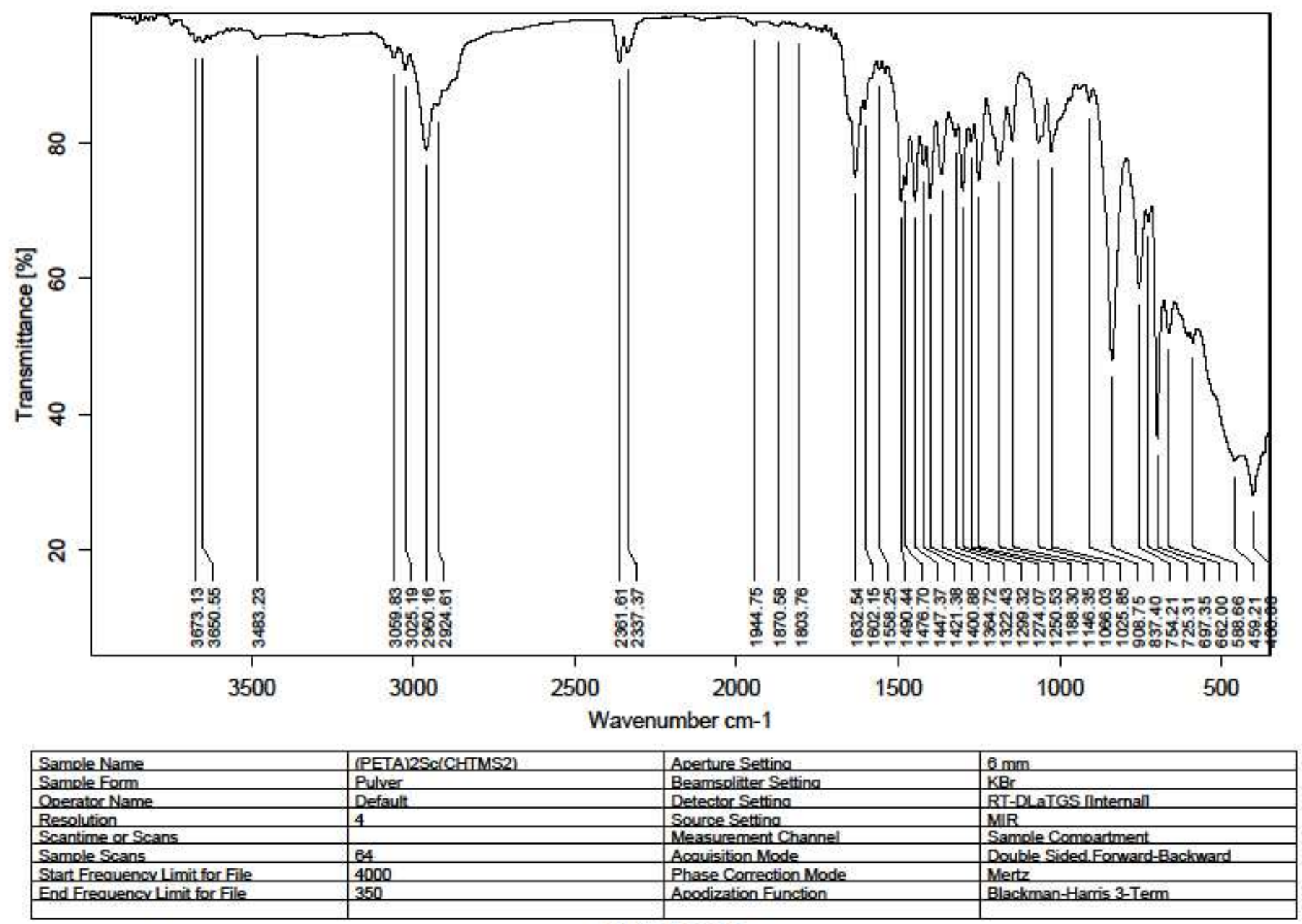

Seite 1 von 2 
Figure S45. IR Spectrum of $\left[\{(S)-\mathrm{PETA}\}_{2} \mathrm{Y}\left\{\mathrm{CH}\left(\mathrm{SiMe}_{3}\right)_{2}\right\}\right](\mathbf{1 0})$.

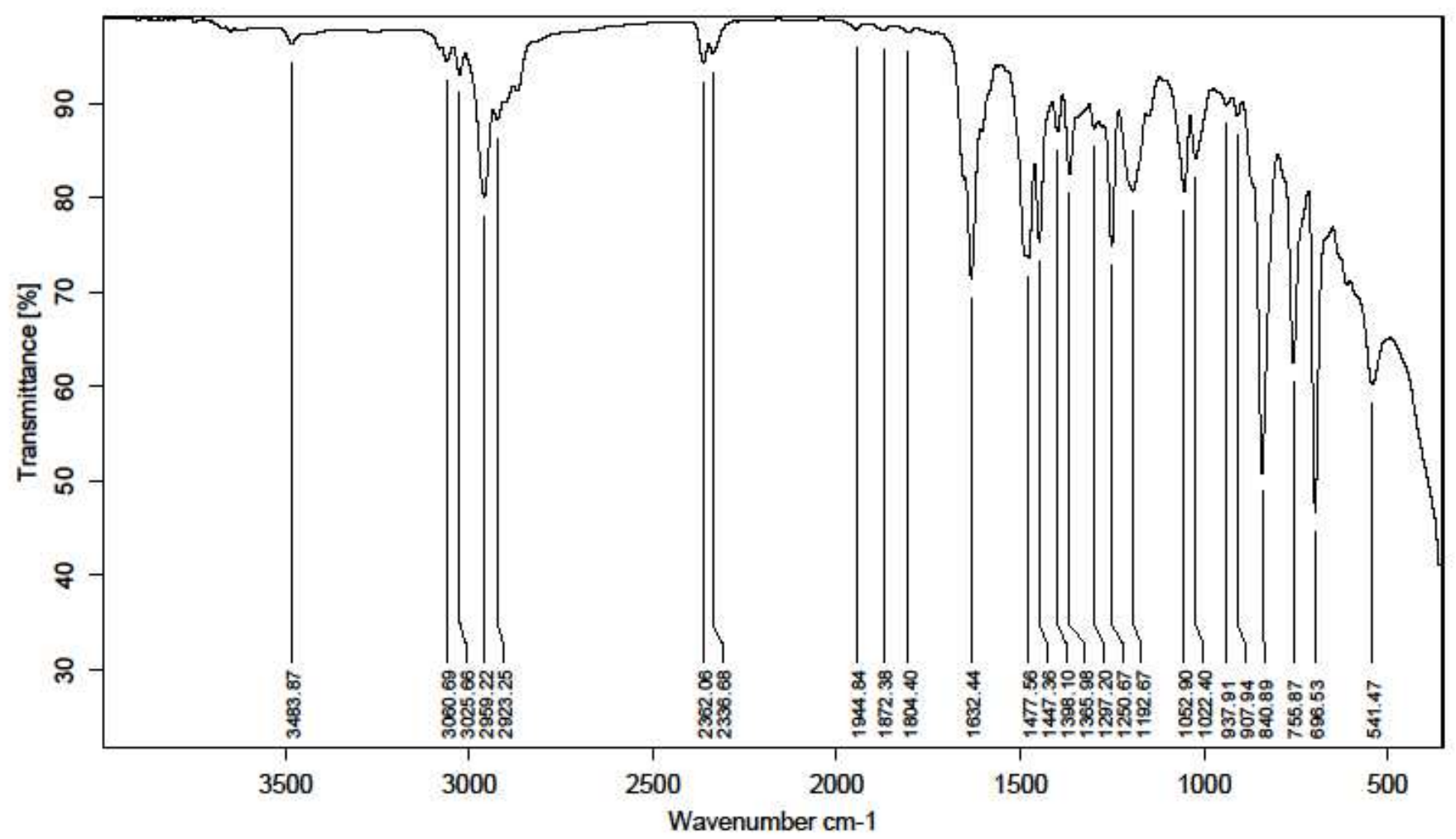

\begin{tabular}{|c|c|c|c|}
\hline Samole Name & (PETA)2Y(CHTMS2) & Aperture Setting & $6 \mathrm{~mm}$ \\
\hline Sample Form & Pulver & Beamsolitter Selting & $\mathrm{KBr}$ \\
\hline Operator Name & Default & Detector Setting & RT-DLaTGS Ilnternall \\
\hline Resolution & 4 & Source Setting & MIR \\
\hline Scantime or Scans & & Measurement Channel & Samole Combartment. \\
\hline Samole Scans & 84 & Acquisition Mode & Double Sided.Forward-Backward \\
\hline Start Freouency L Limit for File & 4000 & Phase Correction Mode & Mertz \\
\hline End Frequency Limit for File & 350 & Acodization Function & Blackman-Harris 3-Term \\
\hline
\end{tabular}

Seite 1 von 2 
Figure S47. IR Spectrum of $\left[\{(S)-\mathrm{PETA}\}_{2} \mathrm{Lu}\left\{\mathrm{CH}\left(\mathrm{SiMe}_{3}\right)_{2}\right\}\right]$ (11).

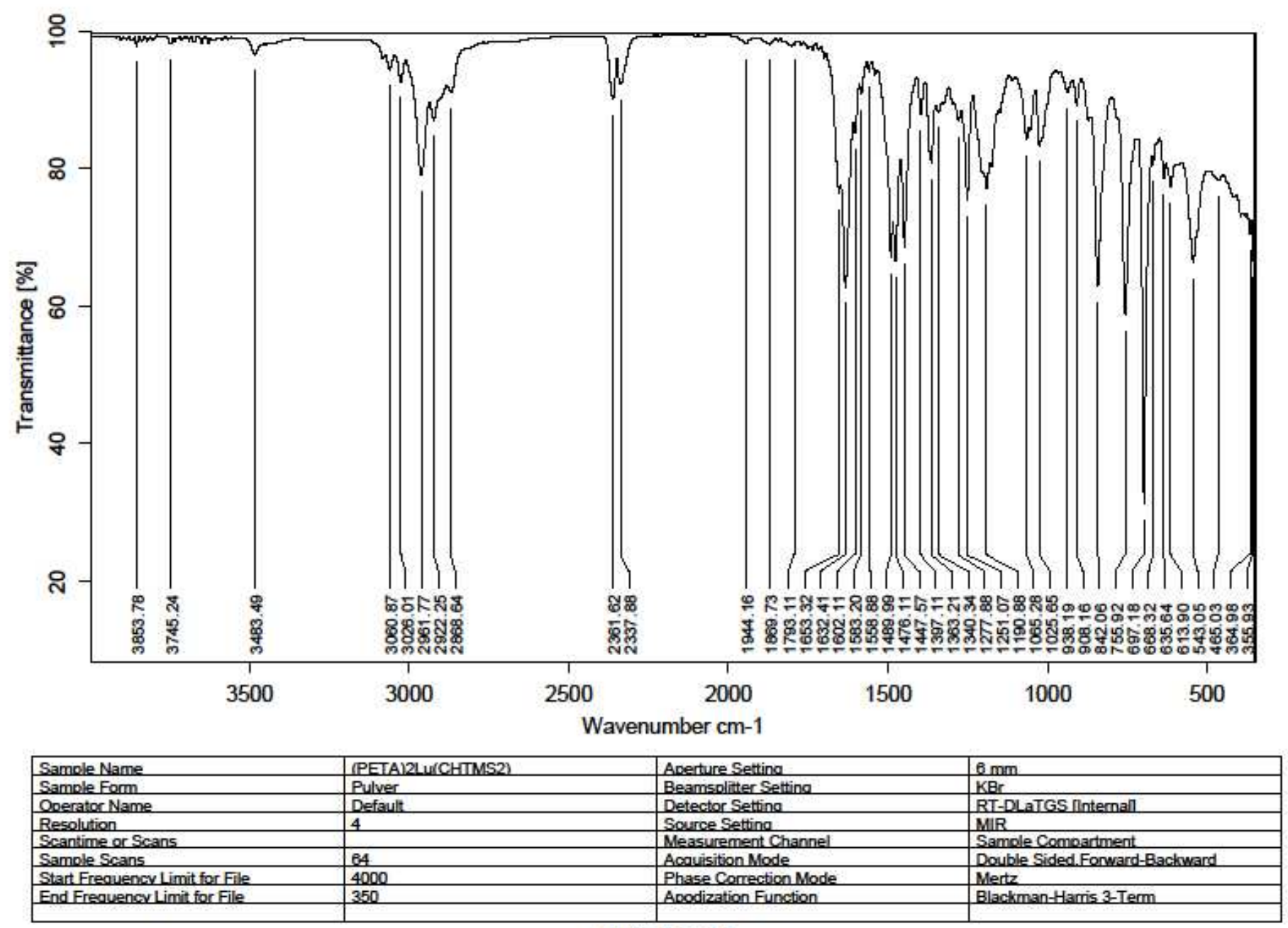

Seite 1 von 2 